\title{
Periodic, quasi-periodic and unbounded solutions of radially symmetric systems with repulsive singularities at resonance
}

\author{
Qihuai Liu, Pedro J. Torres and Dingbian Qian
}

\begin{abstract}
In this paper, we are concerned with periodic solutions, quasiperiodic solutions and unbounded solutions for radially symmetric systems with singularities at resonance, which are $2 \pi$-periodic in time. The method is based on the qualitative analysis of Poincaré map with action-angle variables. The existence of infinitely many periodic and quasiperiodic solutions or unbounded motions depends on the oscillatory properties of a certain function.
\end{abstract}

Mathematics Subject Classification. 34C25, 34B15.

Keywords. Resonance, Unbounded solution, Quasi-periodic solution, Periodic solution, Isochronous system.

\section{Introduction}

Second differential equation with singularities, as an important class of models in non-smooth dynamical systems, has been studied by many researchers, involving the existence and multiplicity of periodic solutions by means of topology degree theory [1-4], Poincaré-Birkhoff twist theorem [5], invariant curves and boundedness of solutions via Moser's small twist theorem [6,7]. The monograph [8] presents an updated review of models with singularities arising in the applied sciences.

Recently, the research of radially symmetric systems with singularities has been a hot topic [9-13]. In this paper, we are concerned with the existence of periodic, quasi-periodic and unbounded solutions of a radially symmetric system in $\mathbb{R}^{N}$ of singular type

$$
\mathbf{u}^{\prime \prime}(t)=-h(|\mathbf{u}(t)|, t) \frac{\mathbf{u}(t)}{|\mathbf{u}(t)|},
$$


where $h:(0,+\infty) \times \mathbb{R} \rightarrow \mathbb{R}$ is a continuous function with a $T$-periodic dependence with respect to the second variable and has a possible singularity at the origin in the first variable. We investigate solutions $\mathbf{u}(t) \in \mathbb{R}^{N}$ which never attain the singularity, in the sense that,

$$
\mathbf{u}(t) \neq 0, \quad \text { for every } t \in \mathbb{R} .
$$

Recently, exploiting the radial symmetry of the system, the existence of periodic solutions of system (1.1) has been proved by means of a topological degree approach [4,9-13]. The repulsive case was studied in [10] for nonlinearities with sublinear growth at infinity, in [13] for nonlinearities with superlinear growth, and in [9] for those with a linearly controlled growth where a non-resonance condition is needed, that is,

$$
\left(\frac{k \pi}{T}\right)^{2}<\alpha \leq \liminf _{x \rightarrow+\infty} \frac{h(x, t)}{x} \leq \limsup _{x \rightarrow+\infty} \frac{h(x, t)}{x} \leq \beta<\left(\frac{(k+1) \pi}{T}\right)^{2}
$$

with some integer $k$ and two constants $\alpha, \beta$.

In this paper, our main interest is to analyze the resonant case and to find out a sufficient condition for the existence of radially $T$-periodic solutions for system (1.1) in case of resonances. For convenience, we fix $T=2 \pi$ from now on. We shall assume that

$$
h(x, t)=a x+f(x, t),
$$

where $f:(0,+\infty) \times \mathbb{R} \rightarrow \mathbb{R}$ is continuous, $2 \pi$-periodic with respect to its second variable, and the resonance condition

$$
a=\frac{n^{2}}{4}, \quad \text { for any integer } n \in \mathbb{N},
$$

holds. Moreover, we assume that

$\left(f_{1}\right)$ The function $f:(0,+\infty) \times \mathbb{R} \rightarrow \mathbb{R}$ is locally Lipschitzian continuous, $T$-periodic and satisfies that

$$
\lim _{x \rightarrow+\infty} f(x, t)=f(+\infty, t)
$$

holds uniformly for $t \in[0,2 \pi]$;

$\left(f_{2}\right)$ there exist some positive constants $M$ and $0<\delta, \nu<1$ such that, for any $x \in(0, \delta)$ and $t \in[0,2 \pi]$,

$$
\left|x^{\nu}\left(f(x, t)+\frac{1}{x^{3}}\right)\right| \leq M
$$

The dynamics of the system is closely related to the function $\sigma_{f}:[0,2 \pi] \rightarrow \mathbb{R}$ defined by

$$
\sigma_{f}(\theta)=\int_{0}^{2 \pi}|\sin (\theta / 2+\sqrt{a} t)| f(+\infty, t) \mathrm{d} t .
$$

Now let us state our main result. 
Theorem 1.1. Assume that $h(x, t)=a x+f(x, t)$ satisfies $(1.3),\left(f_{1}\right)$ and $\left(f_{2}\right)$.

(i) If the function $\sigma_{f}(\theta)$ has no zeros, i.e.,

$$
\sigma_{f}(\theta) \neq 0, \quad \theta \in[0,2 \pi]
$$

then there exist infinitely many integers $p, q$ with $(q, p)=1$, such that system (1.1) has periodic solutions $\mathbf{u}_{q p}(t)$ with minimal period $2 p \pi$, rotating exactly $q$ times around the origin in the period time $2 p \pi$. Moreover, for any $\omega \in \mathbb{R} \backslash \mathbb{Q}$ such that $\omega \ll 1$, system (1.1) has at least one quasi-periodic solution with frequencies $\langle 1, \omega\rangle$.

Furthermore, for any $\mu_{1}, \mu_{2}$ close to zero, there is a constant $B>0$ independent of $q, p$ such that, all those periodic $2 p \pi$-solutions $\mathbf{u}_{q p}(t ; \mu)$ with the angular momentum $\mu \in\left[\mu_{1}, \mu_{2}\right]$ satisfy that

$$
\frac{1}{B\left(\mu_{1}, \mu_{2}\right)}<\left|\mathbf{u}_{q p}(t ; \mu)\right|<B\left(\mu_{1}, \mu_{2}\right), \quad \text { for every } t \in \mathbb{R} .
$$

(ii) Assume that $\sigma_{f}(\theta)$ has at least one zero $\theta_{0}$ and for all $\theta \in[0,2 \pi]$, $\left|\sigma_{f}(\theta)\right|+\left|\sigma_{f}^{\prime}(\theta)\right|>0$. Then,

1. if $\sigma_{f}^{\prime}\left(\theta_{0}\right)>0$, there exists $\lambda_{0}>0$ such that, for $|\mathbf{u}(0)| \geq \lambda_{0}$, the solution $\mathbf{u}(t)$ of system (1.1) satisfies

$$
\lim _{j \rightarrow+\infty}\left|\mathbf{u}\left(t_{j}\right)\right|=+\infty
$$

for some sequence $\left\{t_{j}\right\}_{j=0}^{n}$ with $\lim _{j \rightarrow+\infty} t_{j}=+\infty$.

2. if $\sigma_{f}^{\prime}\left(\theta_{0}\right)<0$, then there exists $\lambda_{0}>0$ such that, for $|\mathbf{u}(0)| \geq \lambda_{0}$, the solution $\mathbf{u}(t)$ of system (1.1) satisfies

$$
\lim _{j \rightarrow-\infty}\left|\mathbf{u}\left(t_{j}\right)\right|=+\infty
$$

for some sequence $\left\{t_{j}\right\}_{j=0}^{n}$ with $\lim _{j \rightarrow+\infty} t_{j}=-\infty$.

The rest of the paper is organized as follows. In Sect. 2, we shall introduce a related scalar singular equation for the radial component that can be regarded as a perturbed isochronous oscillator. Then, an auxiliary result (Theorem 2.1) is stated, which may be of independent interest for the theory of scalar singular equations. Its proof will be the key point for the proof of the main result stated above. After introducing action and angle variables in Sect. 3, we derive an expression for the Poincaré map of (2.12) in Sect. 4 and complete the proof in Sect. 5. Two auxiliary lemmas involving some technical computations are placed separately at the end of the paper.

\section{A related singular equation for the radial component}

The idea of the proof of Theorem 1.1 is to split the system into its radially and angular components and to consider the scalar angular momentum as a parameter. Since system (1.1) is radially symmetric, the orbit of a solution lies on a plane (see, e.g., [4, Appendix A]), so it is possible assume, without loss 
of generality, that $N=2$. Then, writing the solutions of system (1.1) in polar coordinates

$$
\mathbf{u}(t)=x(t) e^{i \theta(t)},
$$

system (1.1) is equivalent to equations

$$
x^{\prime \prime}(t)-\frac{\mu^{2}}{x^{3}(t)}+a x(t)+f(x(t), t)=0,
$$

and

$$
x^{2}(t) \theta^{\prime}(t)=\mu
$$

where $\mu$ is the scalar angular momentum of $\mathbf{u}(t)$, which remains constant along solutions, cf. $[4,14]$. When considering a solution of system (1.1), we will always implicitly assume that $\mu>0$ and $x>0$, so (1.2) is automatically satisfied.

A solution $u: \mathbb{R} \rightarrow \mathbb{R}^{2} \backslash\{0\}$ of (1.1) is said to be radially $T$-periodic if the radial component $x(t)$ is $T$-periodic. In this case, the number $\omega=\frac{\theta(T)-\theta(0)}{T}$ can be interpreted as the average angular speed of $u$ and will be called the rotation number of $u$ and denoted by $\omega=\operatorname{rot} u$. Then, a radially $T$-periodic solution $u$ is $T$-periodic if and only if rot $u$ is an integer multiple of $2 \pi / T$. If rot $u=(m / n)(2 \pi / T)$ for some relatively prime integers $m \neq 0 \neq n$, then $u$ will be a subharmonic with minimal period $n T$. In other case, $u$ is quasiperiodic with two natural frequencies.

As a matter of fact, (2.9) not only models non-zero angular momentum motions in the rotationally symmetric two-dimensional harmonic oscillator [15], but it is also related to other relevant problems arising in the applied sciences, like radially symmetric systems in celestial mechanics [16,17], and Bose-Einstein condensates systems in quantum physics [18].

Equation (2.9) can be regarded as the perturbation of an isochronous system. The second order differential equation

$$
x^{\prime \prime}+V^{\prime}(x)=0
$$

is called an isochronous system if there exist constants $x_{0}, T>0$ and a continuous differentiable function $V$ with $V^{\prime}\left(x_{0}\right)=0,\left(x-x_{0}\right) V^{\prime}(x)>0$, for $x \neq x_{0}$, such that every solution is periodic with periodic $T$. Obviously, the nonlinear equation

$$
x^{\prime \prime}+a x-\frac{\mu^{2}}{x^{3}}=0
$$

is an isochronous system. It is not difficult to show that all solutions are $\pi / \sqrt{a}$-periodic and the least positive period $T=\pi / \sqrt{a}$ is independent of the adjustable parameter $\mu$.

In [19], Bonheure, Fabry and Smets study the forced isochronous oscillators with jumping nonlinearities and oscillators with a repulsive singularity. The results show that if $f(x, t)=g(x)-p(t)$ satisfies that $g(x)$ is bounded and $\lim _{x \rightarrow+\infty} g(x)=g(+\infty)$ exists, then the condition of Lazer-Landesman type:

$$
4 g(+\infty)>\max _{\theta} \int_{0}^{2 \pi}|\sin (n t / 2)| p\left(t+\frac{\theta}{n}\right) \mathrm{d} t
$$


guarantees the existence of $2 \pi$-periodic solutions of (2.9) with $a=n^{2} / 4$. In this case, unbounded solutions are also treated in [20,21]. Recently, Liu [7] proved the boundedness of solutions with the bounded perturbation $g(x)$ under the condition (2.11) of Lazer-Landesman type and other smoothness conditions via Moser's twist theorem. Some analogous problems were considered by many authors for perturbed asymmetric or harmonic isochronous oscillators (e.g. [22-28]). We mention that all these previous results depend on the boundedness of $f$, however, in our assumptions $f$ may be unbounded at the origin.

Without loss of generality, for $\mu \neq 0$, we take $\mu=1$. Otherwise, replacing $x$ with $\sqrt{\mu} x$, and performing a shift to $(2.9)$, then we get

$$
x^{\prime \prime}+a\left(x+c_{0}\right)-\frac{1}{\left(x+c_{0}\right)^{3}}+\frac{1}{\sqrt{\mu}} f\left(\sqrt{\mu}\left(x+c_{0}\right), t\right)=0,
$$

and

$$
\left(x(t)+c_{0}\right)^{2} \varphi^{\prime}(t)=1,
$$

where $c_{0}=a^{-\frac{1}{4}}$. For convenience, we restate the notation of $\frac{1}{\sqrt{\mu}} f(\sqrt{\mu} x, t)$ by $f(x, t)$ so that $\left(2.9^{\prime}\right)$ becomes that

$$
x^{\prime \prime}+a\left(x+c_{0}\right)-\frac{1}{\left(x+c_{0}\right)^{3}}+f\left(\left(x+c_{0}\right), t\right)=0 .
$$

We assume that

$\left(f_{3}\right)$ there exist some positive constants $M$ and $0<\delta, \nu<1$ such that, for any $x \in(0, \delta)$ and $t \in[0,2 \pi]$,

$$
\left|x^{\nu} f(x, t)\right| \leq M \text {. }
$$

Now we are ready to state the following result.

Theorem 2.1. Assume that the function $f$ satisfies assumptions $\left(f_{1}\right)$ and $\left(f_{3}\right)$, and the resonant condition (1.3) holds. Then,

(i) if the function $\sigma_{f}(\theta)$ has no zeros, i.e.,

$$
\sigma_{f}(\theta) \neq 0, \quad \theta \in[0,2 \pi]
$$

holds, then (2.9) has at least one $2 \pi$-periodic solution;

(ii) if the function $\sigma_{f}(\theta)$ has a zero $\theta_{0}$ and for all $\theta \in[0,2 \pi],\left|\sigma_{f}(\theta)\right|+\left|\sigma_{f}^{\prime}(\theta)\right|>$ 0. Furthermore, the following conditions hold:

1. if $\sigma_{f}^{\prime}\left(\theta_{0}\right)>0$, then there exists $\lambda_{0}>0$ such that, for $x_{0}^{2}+y_{0}^{2} \geq \lambda_{0}$, the solution $x(t)$ of $(2.9)$ with $x(0)=x_{0}, x^{\prime}(0)=y_{0}$ satisfies

$$
\lim _{t \rightarrow+\infty}\left(x(t)^{2}+x^{\prime}(t)^{2}+x^{\prime}(t)^{-2}\right)=+\infty .
$$

Moreover, there exits a sequence $\left\{t_{j}\right\}_{j=0}^{n}$ with $\lim _{j \rightarrow+\infty} t_{j}=+\infty$ such that

$$
\lim _{j \rightarrow+\infty}\left(\left|x\left(t_{j}\right)\right|+\left|x^{\prime}\left(t_{j}\right)\right|\right)=+\infty .
$$


2. if $\sigma_{f}^{\prime}\left(\theta_{0}\right)<0$, then there exists $\lambda_{0}>0$ such that, for $x_{0}^{2}+y_{0}^{2} \geq \lambda_{0}$, the solution $x(t)$ of $(2.9)$ with $x(0)=x_{0}, x^{\prime}(0)=y_{0}$ satisfies

$$
\lim _{t \rightarrow-\infty}\left(x(t)^{2}+x^{\prime}(t)^{2}+x^{\prime}(t)^{-2}\right)=+\infty .
$$

Moreover, there exits a sequence $\left\{t_{j}\right\}_{j=0}^{n}$ with $\lim _{j \rightarrow+\infty} t_{j}=-\infty$ such that

$$
\lim _{j \rightarrow-\infty}\left(\left|x\left(t_{j}\right)\right|+\left|x^{\prime}\left(t_{j}\right)\right|\right)=+\infty .
$$

Remark 2.1. In case of $f(x, t)=g(x)-p(t)$ with the limit $\lim _{x \rightarrow+\infty} g(x)=g(+\infty)$ and boundedness of $g$, the condition (2.11) of Lazer-Landesman type implies that (2.13) holds, that is, $\sigma_{p}(\theta) \neq 0$. In fact, let $\tau=t+\frac{\theta}{n}$, then

$$
\begin{aligned}
\int_{0}^{2 \pi} p(t)|\sin (n t / 2+\theta / 2)| \mathrm{d} t & =\int_{\frac{\theta}{n}}^{2 \pi+\frac{\theta}{n}}|\sin (n \tau / 2)| p\left(\tau-\frac{\theta}{n}\right) \mathrm{d} t \\
& =\int_{0}^{2 \pi}|\sin (n \tau / 2)| p\left(\tau-\frac{\theta}{n}\right) \mathrm{d} t
\end{aligned}
$$

Therefore, it follows that

$$
\begin{aligned}
\sigma_{p}(\theta) & =4 g(+\infty)-\int_{0}^{2 \pi} p(t)|\sin (n t / 2+\theta / 2)| \mathrm{d} t \\
& \geq 4 g(+\infty)-\max _{\theta} \int_{0}^{2 \pi}|\sin (n t / 2)| p\left(t+\frac{\theta}{n}\right) \mathrm{d} t>0 .
\end{aligned}
$$

In this case, the results proved by Liu [7] show that there exist infinitely many invariant curves with arbitrarily large amplitude, which implies the boundedness of the solutions of (2.9). On the contrary, Theorem 2.1 shows that when the condition (2.11) of Lazer-Landesman type is lost, all large solutions of (2.9) may be unbounded.

We finish this section by observing that Theorem 2.1 may have an independent interest on the framework of scalar differential equations with singularities.The following illustrative examples are direct applications.

Example 2.1. For any constants $\mu$ and $\nu \in(0,1)$, we can apply Theorem 2.1 to the following equation

$$
x^{\prime \prime}+\frac{1}{4} x-\frac{\mu^{2}}{x^{3}} \pm \frac{1}{x^{\nu}}+\frac{\lambda}{\pi} \arctan x=\frac{(x+\ln x) \sin t}{1+x} .
$$

It is not difficult to verify that if $|\lambda|>1 / 3$, then (2.14) has a $2 \pi$-periodic solution; if $|\lambda|<1 / 3$, then for all large solutions are unbounded in the future or in the past.

Example 2.2. All large solutions of the following equation

$$
x^{\prime \prime}+\frac{1}{4} x-\frac{1}{x^{3}}=\sin t .
$$

are unbounded. 


\section{Construction of action and angle variables}

We carry out the standard reduction for Eq. (2.12) to the action and angle variables [14]. In order to introduce action and angle variables, we consider the auxiliary autonomous system

$$
x^{\prime}=y, \quad y^{\prime}=-a\left(x+c_{0}\right)+\frac{1}{\left(x+c_{0}\right)^{3}} .
$$

For each $\hat{h} \in(0,+\infty)$,

$$
\Gamma: \frac{1}{2} y^{2}+\frac{1}{2} a\left(x+c_{0}\right)^{2}+\frac{1}{2\left(x+c_{0}\right)^{2}}=\hat{h}+\sqrt{a}
$$

defines a simple closed curve in the half plane $\left(-c_{0},+\infty\right)$. is,

Now we define the function $I:(0,+\infty) \rightarrow \mathbb{R}$ by $I(\hat{h})=\frac{1}{2 \pi} \oint_{\Gamma} y \mathrm{~d} x$, that

$$
I(\hat{h})=\frac{1}{\pi} \int_{x_{-}}^{x_{+}} \sqrt{2(\hat{h}+\sqrt{a})-a\left(x+c_{0}\right)^{2}-\left(x+c_{0}\right)^{-2}} \mathrm{~d} x,
$$

where

$$
x_{ \pm}=-c_{0}+\sqrt{a^{-1}\left(\hat{h}+\sqrt{a} \pm \sqrt{\hat{h}^{2}+2 \sqrt{a} \hat{h}}\right)}, \quad \text { for } h \in(0,+\infty) .
$$

Generally, $I$ is called the action of Hamiltonian system on the period annulus. The value of the function $I$ is normalized area of the region in the phase space enclosed by the periodic orbit $\Gamma$. Using Lemma 4.1 in the Appendix, $I(\hat{h})$ can be calculated in a simple implicit form

$$
I(\hat{h})=\frac{\hat{h}}{2 \sqrt{a}} .
$$

Then for every $(x, y) \in\left(-c_{0},+\infty\right) \times \mathbb{R}$, the action and angle variables can be defined by

$$
\begin{aligned}
\tilde{\theta}(x, y) & = \begin{cases}\int_{x_{-}}^{x} \frac{2 \sqrt{a}}{\sqrt{2(h+\sqrt{a})-a\left(\tau+c_{0}\right)^{2}-\left(\tau+c_{0}\right)^{-2}}} \mathrm{~d} \tau, & \text { if } y \geq 0, \\
2 \pi-\int_{x_{-}}^{x} \frac{2 \sqrt{a}}{\sqrt{2(h+\sqrt{a})-a\left(\tau+c_{0}\right)^{2}-\left(\tau+c_{0}\right)^{-2}}} \mathrm{~d} \tau, & \text { if } y<0,\end{cases} \\
& = \begin{cases}\frac{\pi}{2}-\arcsin \frac{h+\sqrt{a}-a\left(x+c_{0}\right)^{2}}{\sqrt{h^{2}+2 \sqrt{a} h}}, & \text { if } y \geq 0, \\
\frac{3 \pi}{2}+\arcsin \frac{h+\sqrt{a}-a\left(x+c_{0}\right)^{2}}{\sqrt{h^{2}+2 \sqrt{a} h}}, & \text { if } y<0,\end{cases}
\end{aligned}
$$

and

$$
\tilde{I}(x, y)=\frac{1}{2 \sqrt{a}} h .
$$

For the convenience of the reader, the calculation of (3.4) is arranged in Appendix B. Here, for simplification of the expression, we omit the independent variables $x, y$ of $h$, and the function $h:\left(-c_{0},+\infty\right) \times \mathbb{R} \rightarrow(0,+\infty)$ is given by

$$
h(x, y)=\frac{1}{2} y^{2}+\frac{a}{2}\left(x+c_{0}\right)^{2}+\frac{1}{2\left(x+c_{0}\right)^{2}}-\sqrt{a} .
$$


Consequently, we have defined the symplectic map

$$
\tilde{\Phi}:\left(-c_{0}, \mathbb{R}\right) \times \mathbb{R} \rightarrow \mathbb{R} / 2 \pi \mathbb{Z} \times(0,+\infty),(x, y) \mapsto(\tilde{\theta}, \tilde{I})
$$

by (3.4) and (3.5), and the associated generating function $\mathcal{G}$ is given by

$$
\mathcal{G}(x, \tilde{I})= \begin{cases}\int_{x_{-}}^{x} \sqrt{2 h(\tilde{I})-a\left(\xi+c_{0}\right)^{2}-\left(\xi+c_{0}\right)^{-2}} \mathrm{~d} \xi, & \text { if } y \geq 0, \\ 2 \pi \tilde{I}-\int_{x_{-}}^{x} \sqrt{2 h(\tilde{I})-a\left(\xi+c_{0}\right)^{2}-\left(\xi+c_{0}\right)^{-2}} \mathrm{~d} \xi, & \text { if } y<0\end{cases}
$$

which satisfies that

$$
\tilde{\theta}=\frac{\partial \mathcal{G}(x, \tilde{I})}{\partial \tilde{I}}, \quad y=\frac{\partial \mathcal{G}(x, \tilde{I})}{\partial x},
$$

where $h(\tilde{I})$ is the inverse function of $\tilde{I}(h)$ defined by (3.3).

In order to simplify the calculations, first we perform a shift of $I$, that is, let $I=\tilde{I}-\frac{1}{2}, \theta=\tilde{\theta}$. In view of (3.4) and together with (3.5) and (3.6), then we have a new symplectic change of variables $\Phi:\left(-c_{0}, \mathbb{R}\right) \times \mathbb{R} \rightarrow \mathbb{R} / 2 \pi \mathbb{Z} \times$ $\left(\frac{1}{2},+\infty\right),(x, y) \mapsto(\theta, I)$ defined by

$$
\begin{aligned}
& x(\theta, I)=\frac{1}{a^{1 / 4}} \sqrt{2 I-\sqrt{4 I^{2}-1} \cos \theta}-c_{0}, \\
& y(\theta, I)=\left(a^{1 / 2}\left(4 I^{2}-1\right) \frac{\left(2 I+\sqrt{4 I^{2}-1} \cos \theta\right)}{4 I^{2} \sin ^{2} \theta+\cos ^{2} \theta}\right)^{\frac{1}{2}} \sin \theta .
\end{aligned}
$$

The deductions of (3.8) and (3.9) are a little long, therefore we place them at the Appendix B. We have examined the Jacobian determinant

$$
\operatorname{det}\left[\frac{\partial(x, y)}{\partial(\theta, I)}\right]=1
$$

which implies that the map $\Phi$ preserves the symplectic form $d x \wedge d y=d \theta \wedge d I$.

The Hamiltonian induced by (2.12) may be written as

$$
\begin{aligned}
\mathrm{H}(x, y, t) & =\frac{1}{2} y^{2}+\frac{a}{2}\left(x+c_{0}\right)^{2}+\frac{1}{2\left(x+c_{0}\right)^{2}}+F\left(x+c_{0}, t\right) \\
& =h(x, y)+F\left(x+c_{0}, t\right),
\end{aligned}
$$

where

$$
F\left(x+c_{0}\right)=\int_{-c_{0}}^{x} f\left(x+c_{0}, t\right) \mathrm{d} x .
$$

In the action and angle variables coordinates $(\theta, I)$ as defined above, the canonically transformed Hamiltonian becomes

$$
\begin{aligned}
\mathrm{H}(\theta, I, t) & =h(x(\theta, I), y(\theta, I))+F\left(x(\theta, I)+c_{0}, t\right) \\
& =\mathrm{H}_{0}(I)+F\left(x(\theta, I)+c_{0}, t\right),
\end{aligned}
$$

where $\mathrm{H}_{0}(I)=2 \sqrt{a} I$ is the unperturbed component of the Hamiltonian. Note that the unperturbed Hamiltonian system with the Hamiltonian $\mathrm{H}_{0}(I)$ is an isochronous system, i.e., each solution of the unperturbed Hamiltonian system is a periodic solution with the least period $\tau=\pi / \sqrt{a}$. 


\section{An expression for the Poincaré map of (2.12)}

In the new coordinates $(\theta, I)$, the Eq. (2.12) with the new Hamiltonian (3.11) has the form

$$
\begin{aligned}
\frac{\mathrm{d} \theta}{\mathrm{d} t} & =\frac{\partial \mathrm{H}}{\partial I}(\theta, I, t) \\
& =2 \sqrt{a}+\frac{a^{-1 / 4}\left(\sqrt{4 I^{2}-1}-2 I \cos \theta\right)}{\sqrt{4 I^{2}-1} \cdot \sqrt{2 I-\sqrt{4 I^{2}-1} \cos \theta}} f\left(\left(x(\theta, I)+c_{0}, t\right),\right. \\
\frac{\mathrm{d} I}{\mathrm{~d} t} & =-\frac{\partial \mathrm{H}}{\partial \theta}(\theta, I, t) \\
& =-\frac{a^{-1 / 4} \sqrt{4 I^{2}-1} \sin \theta}{2 \sqrt{2 I-\sqrt{4 I^{2}-1}} \cos \theta} f\left(\left(x(\theta, I)+c_{0}, t\right) .\right.
\end{aligned}
$$

Denote by $\left(\theta\left(t, \theta_{0}, I_{0}\right), I\left(t, \theta_{0}, I_{0}\right)\right)$ the solution of (4.1) and (4.2) satisfying the initial condition $\theta(0)=\theta_{0}, I(0)=I_{0}$ with $x_{0}=x\left(\theta_{0}, I_{0}\right), y_{0}=y\left(\theta_{0}, I_{0}\right)$.

Now we fix the positive constant $\delta_{0}=\min \left\{\delta, 10^{-4}, \frac{1}{2} a^{-1 / 4}\right\}$. First we have the rough estimates for the derivatives of $\theta$ and $I^{\frac{1}{2}}$ on some intervals in the following lemmas.

Lemma 4.1. Assume that the continuous function $f:\left(-c_{0},+\infty\right) \times[0,2 \pi] \rightarrow \mathbb{R}$ satisfies $\left(f_{1}\right)$ and $\left(f_{2}\right)$, then we have the estimate

$$
\left|\frac{\mathrm{d} \theta}{\mathrm{d} t}-2 \sqrt{a}\right| \leq \frac{4 M_{0} \delta_{0}^{1-\nu}}{\sqrt{4 I^{2}-1}}
$$

for the time $t$ such that

$$
t \in \Omega_{0}:=\left\{t: \frac{2 I(t)-\sqrt{a} \delta_{0}^{2}}{\sqrt{4 I^{2}(t)-1}}<\cos \theta(t) \leq 1 \text { and } I(t)>\frac{1}{\sqrt{a} \delta_{0}^{2}}\right\} .
$$

Proof. If $t \in \Omega_{0}$, then

$$
\cos \theta>\frac{2 I-\sqrt{a} \delta_{0}^{2}}{\sqrt{4 I^{2}-1}} \geq \frac{2 I-\frac{1}{4}}{\sqrt{4 I^{2}-1}} \geq \frac{1}{2}
$$

and we can verify that $x+c_{0}=a^{-1 / 4} \sqrt{2 I-\sqrt{4 I^{2}-1} \cos \theta}<\delta_{0} \leq \delta$. By the condition $\left(f_{2}\right)$, we have

$$
\left|\left(x(\theta, I)+c_{0}\right) f\left(x(\theta, I)+c_{0}, t\right)\right| \leq M_{0}, \quad t \in \Omega_{0} .
$$

From (4.1), we have 


$$
\begin{aligned}
\frac{\mathrm{d} \theta}{\mathrm{d} t}= & 2 \sqrt{a}+\frac{\sqrt{4 I^{2}-1}-2 I \cos \theta}{\sqrt{4 I^{2}-1} \cdot\left(2 I-\sqrt{4 I^{2}-1} \cos \theta\right)} \cdot\left(x(\theta, I)+c_{0}\right) f\left(x(\theta, I)+c_{0}, t\right) \\
= & 2 \sqrt{a}+\frac{2 I+\sqrt{4 I^{2}-1} \cos \theta}{\sqrt{4 I^{2}-1} \cdot\left(\sqrt{4 I^{2}-1}+2 I \cos \theta\right)} \\
& \cdot \frac{4 I^{2} \sin ^{2} \theta-1}{4 I^{2} \sin ^{2} \theta+\cos ^{2} \theta}\left(x(\theta, I)+c_{0}\right) f\left(x(\theta, I)+c_{0}, t\right) \\
= & 2 \sqrt{a}+\frac{2 I+\sqrt{4 I^{2}-1} \cos \theta}{\sqrt{4 I^{2}-1} \cdot\left(\sqrt{4 I^{2}-1}+2 I \cos \theta\right)} \\
& \cdot\left[\frac{\left(4 I^{2}-1\right) \sin ^{2} \theta}{4 I^{2} \sin ^{2} \theta+\cos ^{2} \theta}+\frac{\sin ^{2} \theta-1}{4 I^{2} \sin ^{2} \theta+\cos ^{2} \theta}\right]\left(x(\theta, I)+c_{0}\right) f\left(x(\theta, I)+c_{0}, t\right) .
\end{aligned}
$$

Using Lemma 5.2 in the Appendix A, and taking $n=1, b=4 I^{2}, x=\cos ^{2} \theta$ and $\lambda=\frac{\left(\sqrt{b}-\sqrt{a} \delta_{0}^{2}\right)^{2}}{b-1}$, we obtain that

$$
\begin{aligned}
\frac{\left(4 I^{2}-1\right) \sin ^{2} \theta}{4 I^{2} \sin ^{2} \theta+\cos ^{2} \theta} & =\frac{\left(4 I^{2}-1\right)\left(1-\cos ^{2} \theta\right)}{4 I^{2}+\left(4 I^{2}-1\right) \cos ^{2} \theta} \\
& \leq \max \{0, f(\lambda)\}=\frac{4 I^{2}-1-\left(2 I-\sqrt{a} \delta_{0}^{2}\right)^{2}}{4 I^{2}-\left(2 I-\sqrt{a} \delta_{0}^{2}\right)^{2}} \leq 1 .
\end{aligned}
$$

On the other hand, we have

$$
\frac{2 I+\sqrt{4 I^{2}-1} \cos \theta}{\sqrt{4 I^{2}-1}+2 I \cos \theta} \leq \frac{4}{3} \text { and } \quad\left|\frac{\sin ^{2} \theta-1}{4 I^{2} \sin ^{2} \theta+\cos ^{2} \theta}\right| \leq 2 .
$$

Therefore, it follows that, for $t \in \Omega_{1}$,

$$
\left|\frac{\mathrm{d} \theta}{\mathrm{d} t}-2 \sqrt{a}\right| \leq \frac{4 M_{0} \delta_{0}^{1-\nu}}{\sqrt{4 I^{2}-1}} .
$$

So we concludes that (4.3) holds.

Lemma 4.2. Assume that the continuous function $f:\left(-c_{0},+\infty\right) \times[0,2 \pi] \rightarrow \mathbb{R}$ satisfies $\left(f_{1}\right)$ and $\left(f_{3}\right)$, then there exists a positive constant $C$ such that

$$
\left|\frac{\mathrm{d} I^{1 / 2}}{\mathrm{~d} t}\right| \leq C:=\frac{a^{-1 / 4}}{2} M_{1}
$$

for $t \in[0,2 \pi] \backslash \Omega_{0}$ with

$$
M_{1}=\sup \left\{|f(x, t)|:(x, t) \in\left[\frac{\delta_{0}}{2},+\infty\right) \times[0,2 \pi]\right\} .
$$

Proof. When $t \in[0,2 \pi] \backslash \Omega_{0}$, if

$$
\cos \theta \leq \frac{2 I-\sqrt{a} \delta_{0}^{2}}{\sqrt{4 I^{2}-1}} \quad \text { and } \quad I \geq \frac{1}{\sqrt{a} \delta_{0}^{2}}
$$


then $x(\theta, I)+c_{0} \geq \delta_{0} \geq \delta_{0} / 2 ;$ if $I \leq 1 /\left(\sqrt{a} \delta_{0}^{2}\right)$, then

$$
\begin{aligned}
x(\theta, I)+c_{0} & \geq a^{-\frac{1}{4}} \sqrt{2 I-\sqrt{4 I^{2}-1}} \\
& \geq \frac{a^{-\frac{1}{4}}}{\sqrt{2 I+\sqrt{4 I^{2}-1}}} \geq \frac{a^{-\frac{1}{4}}}{\sqrt{4 I}} \geq \frac{\delta_{0}}{2} .
\end{aligned}
$$

In view of (4.2), we have that

$$
\frac{\mathrm{d} I^{\frac{1}{2}}}{\mathrm{~d} t}=-\frac{a^{-1 / 4} \sqrt{4 I^{2}-1} \sin \theta}{4 \sqrt{I} \cdot \sqrt{2 I-\sqrt{4 I^{2}-1} \cos \theta}} f\left(x(\theta, I)+c_{0}, t\right) .
$$

Note that

$$
\begin{aligned}
\frac{\left|\sqrt{4 I^{2}-1} \sin \theta\right|}{2 \sqrt{I} \cdot \sqrt{2 I-\sqrt{4 I^{2}-1} \cos \theta}} & =\frac{\left|\sqrt{4 I^{2}-1} \sin \theta\right| \cdot \sqrt{2 I+\sqrt{4 I^{2}-1} \cos \theta}}{2 \sqrt{I} \cdot \sqrt{4 I^{2} \sin ^{2} \theta+\cos ^{2} \theta}} \\
& \leq \frac{\left|\sqrt{4 I^{2}-1} \sin \theta\right| \cdot \sqrt{2 I+\sqrt{4 I^{2}-1} \cos \theta}}{2 \sqrt{I} \cdot \sqrt{\left(4 I^{2}-1\right) \sin ^{2} \theta}} \\
& =\frac{\sqrt{2 I+\sqrt{4 I^{2}-1} \cos \theta}}{2 \sqrt{I}} \leq 1 .
\end{aligned}
$$

Consequently, by (4.6) we have the desired inequality (4.5).

Lemma 4.3. Assume that the continuous function $f:\left(-c_{0},+\infty\right) \times[0,2 \pi] \rightarrow \mathbb{R}$ satisfies $\left(f_{1}\right)$ and $\left(f_{3}\right)$. Then the solution $(\theta, I)$ of $(4.1)$ and (4.2) satisfies that

$$
\begin{aligned}
I(t)^{\frac{1}{2}} & =I_{0}^{\frac{1}{2}}+O(1), \quad t \in[0,2 \pi], I_{0} \rightarrow+\infty, \\
\theta(t) & =\theta_{0}+2 \sqrt{a} t+O\left(I_{0}^{-\frac{1}{2}}\right), \quad t \in[0,2 \pi] \quad \text { and } \quad \theta_{0} \in \mathbb{R}, I_{0} \rightarrow+\infty,
\end{aligned}
$$

where $O(1)$ denotes a bounded quantity which is independent of $I_{0}$, and $O\left(I_{0}^{-1 / 2}\right)$ denotes an infinitesimal of the same order as $I_{0}^{-1 / 2}$.

Proof. First, we claim that

$$
I(t)^{\frac{1}{2}}=I_{0}^{\frac{1}{2}}+O(1), \quad t \in \Omega_{0}, I_{0} \rightarrow+\infty .
$$

If $t \in \Omega_{0}$, then we can verify that

$$
x+c_{0}=a^{-1 / 4} \sqrt{2 I-\sqrt{4 I^{2}-1} \cos \theta}<\delta_{0} \leq \delta .
$$

Form (4.6), we have

$$
\begin{aligned}
\frac{\mathrm{d} I^{\frac{1}{2}}}{\mathrm{~d} t} & =-\frac{a^{-1 / 4} \sqrt{4 I^{2}-1} \sin \theta}{4 \sqrt{I} \cdot \sqrt{2 I-\sqrt{4 I^{2}-1} \cos \theta}} f\left(x(\theta, I)+c_{0}, t\right) \\
& =-\frac{\sqrt{4 I^{2}-1} \sin \theta}{4 \sqrt{I}\left(2 I-\sqrt{4 I^{2}-1} \cos \theta\right)} \cdot\left(x(\theta, I)+c_{0}\right) f\left(x(\theta, I)+c_{0}, t\right) .
\end{aligned}
$$


Then it follows that

$$
\begin{aligned}
I^{-\frac{1}{2}} \frac{\mathrm{d} I^{\frac{1}{2}}}{\mathrm{~d} t}= & -\frac{\sqrt{4 I^{2}-1} \sin \theta}{4 I\left(2 I-\sqrt{4 I^{2}-1} \cos \theta\right)} \cdot\left(x(\theta, I)+c_{0}\right) f\left(x(\theta, I)+c_{0}, t\right) \\
= & -\frac{2 I+\sqrt{4 I^{2}-1} \cos \theta}{4 I} \cdot \frac{\sqrt{4 I^{2}-1} \sin \theta}{4 I^{2}-\left(4 I^{2}-1\right) \cos ^{2} \theta} \\
& \cdot\left(x(\theta, I)+c_{0}\right) f\left(x(\theta, I)+c_{0}, t\right) .
\end{aligned}
$$

Again, using Lemma 5.2 and taking $n=2, b=4 I^{2}, x=\cos ^{2} \theta$ and $\lambda=$ $\frac{\left(\sqrt{b}-\sqrt{a} \delta_{0}^{2}\right)^{2}}{b-1}$, we obtain that

$$
\begin{aligned}
\left(\frac{\sqrt{4 I^{2}-1} \sin \theta}{4 I^{2}-\left(4 I^{2}-1\right) \cos ^{2} \theta}\right)^{2} & =\frac{\left(4 I^{2}-1\right)\left(1-\cos ^{2} \theta\right)}{\left(4 I^{2}-\left(4 I^{2}-1\right) \cos ^{2} \theta\right)^{2}} \leq \max \left\{\frac{1}{4}, f(\lambda)\right\} \\
& =\max \left\{\frac{1}{4}, \frac{4 I^{2}-1-\left(2 I-\sqrt{a} \delta_{0}^{2}\right)^{2}}{\left(4 I^{2}-\left(2 I-\sqrt{a} \delta_{0}^{2}\right)^{2}\right)^{2}}\right\} \\
& \leq \max \left\{\frac{1}{4}, \frac{1}{4 I^{2}}\right\} \leq 1 .
\end{aligned}
$$

Therefore, we obtain

$$
\left|I^{-\frac{1}{2}} \frac{\mathrm{d} I^{\frac{1}{2}}}{\mathrm{~d} t}\right| \leq M_{0} \delta_{0}^{1-\nu}
$$

which yields that

$$
\left|\ln I(t)^{\frac{1}{2}}-\ln I_{0}^{\frac{1}{2}}\right| \leq M_{0} \delta_{0}^{1-\nu} \int_{\Omega_{0}} \mathrm{~d} t \leq 2 \pi M_{0} \delta_{0}^{1-\nu} .
$$

Then we get that, for $t \in \Omega_{0}$,

$$
I_{0}^{-\frac{1}{2}} \exp \left(-2 \pi M_{0} \delta_{0}^{1-\nu}\right) \leq I(t)^{-\frac{1}{2}} \leq I_{0}^{-\frac{1}{2}} \exp \left(2 \pi M_{0} \delta_{0}^{1-\nu}\right) .
$$

At the same time, when $t \in \Omega_{0}$, we note that $\cos \theta>\frac{2 I-\sqrt{a} \delta_{0}^{2}}{\sqrt{4 I^{2}-1}}=\frac{1-\frac{\sqrt{a} \delta_{0}^{2}}{2 I}}{\sqrt{1-\frac{1}{4 I^{2}}}} \geq 1-\frac{\sqrt{a} \delta_{0}^{2}}{2 I} \geq 1-\frac{\sqrt{a} \delta_{0}^{2}}{2} \exp \left(4 \pi M_{0} \delta_{0}^{1-\nu}\right) \cdot \frac{1}{I_{0}}$.

By Lemma 4.2, we have

$$
\frac{\mathrm{d} \theta}{\mathrm{d} t} \geq 2 \sqrt{a}-\frac{2 M_{0}}{I_{0}} \exp \left(4 \pi M_{0} \delta_{0}^{1-\nu}\right) \geq \sqrt{a},
$$

for $I_{0}$ large enough.

Consequently, for $I_{0}$ large enough, we have that

$$
\begin{aligned}
\operatorname{Meas}\left(\Omega_{0}\right) & \leq \frac{2}{\sqrt{a}} \arccos \left(1-\frac{\sqrt{a} \delta_{0}^{2}}{2} \exp \left(4 \pi M_{0} \delta_{0}^{1-\nu}\right) \cdot \frac{1}{I_{0}}\right) \\
& \leq \frac{2 \sqrt{2} \delta_{0}}{a^{\frac{1}{4}}} \exp \left(2 \pi M_{0} \delta_{0}^{1-\nu}\right) I_{0}^{-\frac{1}{2}}
\end{aligned}
$$


where the second inequality is obtained by the inequality $\arccos (1-x) \leq$ $2 \sqrt{x}, x \in[0,1]$. Now returning to $(4.9)$, we will give a more concise estimate for $I(t)$ when $t \in \Omega_{0}$. So it follows form (4.9) that

$$
\left|\ln I(t)^{\frac{1}{2}}-\ln I_{0}^{\frac{1}{2}}\right| \leq M_{0} \delta_{0}^{1-\nu} \int_{\Omega_{0}} \mathrm{~d} t \leq \frac{2 \sqrt{2} M_{0} \delta_{0}^{2-\nu}}{a^{\frac{1}{4}}} \exp \left(2 \pi M_{0} \delta_{0}^{1-\nu}\right) I_{0}^{-\frac{1}{2}} .
$$

Then

$$
I(t)^{\frac{1}{2}}=I_{0}^{\frac{1}{2}} \exp \left[O\left(I_{0}^{-\frac{1}{2}}\right)\right]=I_{0}^{\frac{1}{2}}+O(1), \quad \text { as } I_{0} \rightarrow+\infty .
$$

When $t \in[0,2 \pi] \backslash \Omega_{0}$, integrating both sides of the Eq. (4.5) with respect to $t$ on the interval $[0, t]$, by Lemma 4.2 we obtain $(4.7)$. Therefore, we have proved the first part of the lemma.

In the following, we will prove the second equality (4.8) in the lemma. In case of $t \in \Omega_{0}$, integrating both sides of the Eq. (4.3) and together with (4.7), we obtain the desired equality (4.8).

When $t \in[0,2 \pi] \backslash \Omega_{0}, x(\theta, I)+c_{0} \geq \delta_{0} \geq \delta_{0} / 2$. Furthermore, by (4.8) we have that

$$
I(t)^{-\frac{1}{2}}=I_{0}^{-\frac{1}{2}}+O\left(I_{0}^{-1}\right), \quad t \in[0,2 \pi], I_{0} \rightarrow+\infty .
$$

Now we claim that

$$
\left|\sqrt{4 I^{2}-1}-2 I \cos \theta\right| \leq 2 \sqrt{I} \sqrt{2 I-\sqrt{4 I^{2}-1} \cos \theta} .
$$

In fact, squaring both sides of (4.12), performing transposition of terms and simplifying, we have that $4 I^{2} \cos ^{2} \theta \leq 4 I^{2}+1$, which holds naturally.

On the other hand, using the equalities (4.11) and (4.12), we have

$$
\begin{aligned}
& \left|\frac{a^{-1 / 4}\left(\sqrt{4 I^{2}-1}-2 I \cos \theta\right)}{\sqrt{4 I^{2}-1} \cdot \sqrt{2 I-\sqrt{4 I^{2}-1} \cos \theta}} f\left(x(\theta, I)+c_{0}, t\right)\right| \\
& \quad \leq \frac{a^{-1 / 4} 2 \sqrt{I}}{\sqrt{4 I^{2}-1}} M_{1} \leq \frac{a^{-1 / 4}}{\sqrt{I}} M_{1} \\
& \quad=O\left(I_{0}^{-\frac{1}{2}}\right), \quad t \in[0,2 \pi] \backslash \Omega_{0}, I_{0} \rightarrow+\infty,
\end{aligned}
$$

where $M_{1}$ is a positive constant defined in Lemma 4.2. Consequently, in view of (4.1) we know that, for $I_{0} \rightarrow+\infty$,

$$
\frac{\mathrm{d} \theta}{\mathrm{d} t}=2 \sqrt{a}+O\left(I_{0}^{-\frac{1}{2}}\right), \quad t \in[0,2 \pi] \backslash \Omega_{0},
$$

which implies the desired equality (4.8).

From Lemma 4.3, the equalities (4.7) and (4.8) imply the global existence of solutions of Eqs. (4.1) and (4.2) with the initial value $\left(\theta_{0}, I_{0}\right)$, when $I_{0}$ is large enough. Therefore, the Poincaré mapping $\mathrm{P}$

$$
\mathrm{P}:\left(\theta_{0}, I_{0}\right) \rightarrow\left(\theta_{1}, I_{1}\right)=\left(\theta\left(2 \pi, \theta_{0}, I_{0}\right), I\left(2 \pi, \theta_{0}, I_{0}\right)\right) .
$$

is well defined. In the following, we will give a scale of solutions for (4.1) and (4.2) when $I_{0}$ is taken for large enough. 
Using Taylor series expansion, together with (4.8) we have that, for any $t \in[0,2 \pi]$ and $\theta_{0} \in \mathbb{R}, \sin \theta=\sin \left(\theta_{0}+2 \sqrt{a} t\right)+O\left(I_{0}^{-\frac{1}{2}}\right)$ as $I_{0} \rightarrow+\infty$. Also, by (4.7), we have

$$
I(t)^{-2}=I_{0}^{-2}+O\left(I_{0}^{-\frac{5}{2}}\right), \quad t \in[0,2 \pi], I_{0} \rightarrow+\infty,
$$

which implies that

$$
\sqrt{4-I^{-2}}=\sqrt{4-I_{0}^{-2}}+O\left(I_{0}^{-\frac{5}{2}}\right), \quad t \in[0,2 \pi], I_{0} \rightarrow+\infty .
$$

Similarly, for any $t \in[0,2 \pi]$,

$$
\sqrt{2-\sqrt{4-I^{-2}} \cos \theta}=\sqrt{2-\sqrt{4-I_{0}^{-2}} \cos \left(\theta_{0}+2 \sqrt{a} t\right)}+O\left(I_{0}^{-\frac{1}{2}}\right), \quad I_{0} \rightarrow+\infty .
$$

Therefore, we know that

$$
\begin{gathered}
\frac{\sqrt{4 I^{2}-1} \sin \theta}{\sqrt{I} \cdot \sqrt{2 I-\sqrt{4 I^{2}-1} \cos \theta}}=\sqrt{4-I^{-2}} \cdot \frac{1}{\sqrt{2-\sqrt{4-I^{-2}} \cos \theta}} \cdot \sin \theta \\
=\frac{\sqrt{4 I_{0}^{2}-1} \sin \left(\theta_{0}+2 \sqrt{a} t\right)}{\sqrt{I_{0}} \cdot \sqrt{2 I_{0}-\sqrt{4 I_{0}^{2}-1} \cos \left(\theta_{0}+2 \sqrt{a} t\right)}}+O\left(I_{0}^{-\frac{1}{2}}\right), \quad I_{0} \rightarrow+\infty,
\end{gathered}
$$

Let $\mathcal{K}\left(I_{0}\right)=\sqrt{1-1 / 4 I_{0}^{2}}$. Then it follows that

$$
\begin{aligned}
\frac{\mathrm{d} I^{\frac{1}{2}}}{\mathrm{~d} t}= & {\left[-\frac{a^{-1 / 4} \sqrt{4 I_{0}^{2}-1} \sin \left(\theta_{0}+2 \sqrt{a} t\right)}{4 \sqrt{I_{0}} \cdot \sqrt{2 I_{0}-\sqrt{4 I_{0}^{2}-1} \cos \left(\theta_{0}+2 \sqrt{a} t\right)}}+O\left(I_{0}^{-\frac{1}{2}}\right)\right] } \\
& \cdot f\left(a^{-1 / 4} \sqrt{2 I_{0}-\sqrt{4 I_{0}^{2}-1} \cos \left(\theta_{0}+2 \sqrt{a} t\right)}+O(1), t\right) \\
= & -\frac{a^{-1 / 4} \mathcal{K}\left(I_{0}\right) \sin \left(\theta_{0}+2 \sqrt{a} t\right)}{2 \sqrt{2} \sqrt{1-\mathcal{K}\left(I_{0}\right) \cos \left(\theta_{0}+2 \sqrt{a} t\right)}} f\left(x\left(\theta_{0}, I_{0}\right)+O(1), t\right) \\
& +O\left(I_{0}^{-\frac{1}{2}}\right) f\left(x\left(\theta_{0}, I_{0}\right)+O(1), t\right), I_{0} \rightarrow+\infty .
\end{aligned}
$$

Integrating both sides of (4.15) over the interval $[0,2 \pi]$ and setting $\gamma=$ $(4 a)^{-1 / 4}$, we obtain

$$
\begin{aligned}
I_{1}^{\frac{1}{2}}= & I_{0}^{\frac{1}{2}}-\gamma \int_{0}^{2 \pi} \frac{\mathcal{K}\left(I_{0}\right) \sin \left(\theta_{0}+2 \sqrt{a} t\right)}{2 \sqrt{1-\mathcal{K}\left(I_{0}\right) \cos \left(\theta_{0}+2 \sqrt{a} t\right)}} \cdot f\left(x\left(\theta_{0}, I_{0}\right)+O(1), t\right) \mathrm{d} t \\
& +\int_{0}^{2 \pi} O\left(I_{0}^{-\frac{1}{2}}\right) f\left(x\left(\theta_{0}, I_{0}\right)+O(1), t\right) \mathrm{d} t, \quad I_{0} \rightarrow+\infty .
\end{aligned}
$$

Similarly, substituting (4.8) into (4.1), we obtain that, for $t \in[0,2 \pi]$ and $I_{0} \rightarrow+\infty$,

$$
\begin{aligned}
\frac{\mathrm{d} \theta}{\mathrm{d} t}= & 2 \sqrt{a}+\left[\gamma I_{0}^{-\frac{1}{2}} \frac{\mathcal{K}\left(I_{0}\right)-\cos \left(\theta_{0}+2 \sqrt{a} t\right)}{\mathcal{K}\left(I_{0}\right) \sqrt{1-\mathcal{K}\left(I_{0}\right) \cos \left(\theta_{0}+2 \sqrt{a} t\right)}}+O\left(I_{0}^{-1}\right)\right] \\
& \cdot f\left(a^{-1 / 4} \sqrt{2 I_{0}} \sqrt{1-\mathcal{K}\left(I_{0}\right) \cos \left(\theta_{0}+2 \sqrt{a} t\right)}+O(1), t\right) .
\end{aligned}
$$


Therefore, we get that, for $I_{0} \rightarrow+\infty$,

$$
\begin{aligned}
\theta_{1}= & \theta_{0}+4 \sqrt{a} \pi+\gamma I_{0}^{-\frac{1}{2}} \int_{0}^{2 \pi} \frac{\mathcal{K}\left(I_{0}\right)-\cos \left(\theta_{0}+2 \sqrt{a} t\right)}{\mathcal{K}\left(I_{0}\right) \sqrt{1-\mathcal{K}\left(I_{0}\right) \cos \left(\theta_{0}+2 \sqrt{a} t\right)}} \\
& \cdot f\left(a^{-1 / 4} \sqrt{2 I_{0}} \sqrt{1-\mathcal{K}\left(I_{0}\right) \cos \left(\theta_{0}+2 \sqrt{a} t\right)}+O(1), t\right) \mathrm{d} t \\
& +\int_{0}^{2 \pi} O\left(I_{0}^{-1}\right) \cdot f\left(a^{-1 / 4} \sqrt{2 I_{0}} \sqrt{1-\mathcal{K}\left(I_{0}\right) \cos \left(\theta_{0}+2 \sqrt{a} t\right)}+O(1), t\right) \mathrm{d} t .
\end{aligned}
$$

Write

$$
\begin{aligned}
\varphi_{1}\left(\theta_{0}, I_{0}, t\right) & =\frac{\mathcal{K}\left(I_{0}\right)-\cos \left(\theta_{0}+2 \sqrt{a} t\right)}{\sqrt{2} \mathcal{K}\left(I_{0}\right) \sqrt{1-\mathcal{K}\left(I_{0}\right) \cos \left(\theta_{0}+2 \sqrt{a} t\right)}}, \\
\varphi_{2}\left(\theta_{0}, I_{0}, t\right) & =\frac{\mathcal{K}\left(I_{0}\right) \sin \left(\theta_{0}+2 \sqrt{a} t\right)}{\sqrt{2} \sqrt{1-\mathcal{K}\left(I_{0}\right) \cos \left(\theta_{0}+2 \sqrt{a} t\right)}}, \\
\psi_{1}\left(\theta_{0}, I_{0}\right) & =\int_{0}^{2 \pi} \varphi_{1}\left(\theta_{0}, I_{0}, t\right) \cdot f\left(x\left(\theta_{0}, I_{0}\right)+O(1), t\right) \mathrm{d} t \\
\psi_{2}\left(\theta_{0}, I_{0}\right) & =\int_{0}^{2 \pi} \varphi_{2}\left(\theta_{0}, I_{0}, t\right) \cdot f\left(x\left(\theta_{0}, I_{0}\right)+O(1), t\right) \mathrm{d} t .
\end{aligned}
$$

Then for $I_{0} \rightarrow+\infty$, we get the Poincaré map

$$
\left\{\begin{array}{c}
\theta_{1}=\theta_{0}+4 \sqrt{a} \pi+a^{-1 / 4} I_{0}^{-\frac{1}{2}} \psi_{1}\left(\theta_{0}, I_{0}\right) \\
\quad+\int_{0}^{2 \pi} O\left(I_{0}^{-1}\right) f\left(x\left(\theta_{0}, I_{0}\right)+O(1), t\right) \mathrm{d} t \\
I_{1}^{\frac{1}{2}}=I_{0}^{\frac{1}{2}}-\frac{1}{2} a^{-1 / 4} \psi_{2}\left(\theta_{0}, I_{0}\right) \\
\quad+\int_{0}^{2 \pi} O\left(I_{0}^{-\frac{1}{2}}\right) f\left(x\left(\theta_{0}, I_{0}\right)+O(1), t\right) \mathrm{d} t .
\end{array}\right.
$$

Lemma 4.4. Assume that the continuous function $f:\left(-c_{0},+\infty\right) \times[0,2 \pi] \rightarrow \mathbb{R}$ satisfies $\left(f_{1}\right)$ and $\left(f_{3}\right)$. Then for $I_{0} \rightarrow+\infty$, we have that

$$
\psi_{1}\left(\theta_{0}, I_{0}\right)=\int_{0}^{2 \pi}\left|\sin \left(\theta_{0} / 2+\sqrt{a} t\right)\right| f(+\infty, t) \mathrm{d} t+o(1)
$$

and

$$
\psi_{2}\left(\theta_{0}, I_{0}\right)=\int_{0}^{2 \pi} \operatorname{sign}\left(\sin \left(\theta_{0} / 2+\sqrt{a} t\right)\right) \cdot \cos \left(\theta_{0} / 2+\sqrt{a} t\right) f(+\infty, t) \mathrm{d} t+o(1)
$$

uniformly with respect to $\theta_{0} \in[0,2 \pi]$, where o(1) denotes an infinitesimal as $I_{0} \rightarrow+\infty$.

Proof. Let us define the sets $\Sigma_{1}\left(\theta_{0}\right), \Sigma_{2}\left(\theta_{0}\right)$ and $\Sigma_{3}\left(\theta_{0}\right)$ by

$$
\begin{aligned}
& \Sigma_{1}\left(\theta_{0}\right)=\left\{t \mid \cos \left(\theta_{0}+2 \sqrt{a} t\right)>\frac{2 I_{0}-\sqrt{a} \delta_{0}^{2}}{\sqrt{4 I_{0}^{2}-1}}, t \in[0,2 \pi]\right\}, \\
& \Sigma_{2}\left(\theta_{0}\right)=\left\{t \mid \frac{2 I_{0}^{2}-\sqrt{a} \delta_{0}^{2}}{\sqrt{4 I_{0}^{2}-1}} \geq \cos \left(\theta_{0}+2 \sqrt{a} t\right) \geq 1-\frac{1}{4 I_{0}^{1 / 2}}, t \in[0,2 \pi]\right\},
\end{aligned}
$$


and

$$
\Sigma_{3}\left(\theta_{0}\right)=[0,2 \pi] \backslash\left(\Sigma_{1}\left(\theta_{0}\right) \cup \Sigma_{2}\left(\theta_{0}\right)\right),
$$

for $I_{0}$ large enough, respectively.

For any $\theta_{0} \in \mathbb{R}, t \in \Sigma_{1}\left(\theta_{0}\right)$ and $I_{0}$ is large enough, using the definition of $\mathcal{K}\left(I_{0}\right)$ and the inequality (4.12) we know that

$$
\begin{aligned}
& \left|\varphi_{1}\left(\theta_{0}, I_{0}, t\right) f\left(x\left(\theta_{0}, I_{0}\right)+O(1), t\right)\right| \\
& \quad \leq\left|\frac{\mathcal{K}\left(I_{0}\right)-\cos \left(\theta_{0}+2 \sqrt{a} t\right)}{\sqrt{2} \mathcal{K}\left(I_{0}\right) \sqrt{1-\mathcal{K}\left(I_{0}\right) \cos \left(\theta_{0}+2 \sqrt{a} t\right)}}\right| \cdot \frac{M_{0}}{\left(\sqrt{2 I_{0}-\sqrt{4 I_{0}^{2}-1} \cos \theta_{0}}+O(1)\right)^{\nu}} \\
& \quad \leq\left|\frac{\sqrt{I_{0}}\left(\sqrt{4 I_{0}^{2}-1}-2 I_{0} \cos \left(\theta_{0}+2 \sqrt{a} t\right)\right)}{\sqrt{4 I_{0}^{2}-1} \cdot \sqrt{2 I_{0}-\sqrt{4 I_{0}^{2}-1} \cos \left(\theta_{0}+2 \sqrt{a} t\right)}}\right| \cdot M_{0} I_{0}^{\frac{\nu}{2}} \\
& \quad \leq \frac{2 M_{0} I_{0}^{1+\frac{\nu}{2}}}{\sqrt{4 I_{0}^{2}-1}} \leq 3 M_{0} I_{0}^{\frac{\nu}{2}} \cdot
\end{aligned}
$$

Thus, we have

$$
\begin{aligned}
& \left|\int_{\Sigma_{1}\left(\theta_{0}\right)} \varphi_{1}\left(\theta_{0}, I_{0}, t\right) f\left(x\left(\theta_{0}, I_{0}\right)+O(1), t\right) \mathrm{d} t\right| \\
& \quad \leq \int_{\Sigma_{1}\left(\theta_{0}\right)}\left|\varphi_{1}\left(\theta_{0}, I_{0}, t\right) f\left(x\left(\theta_{0}, I_{0}\right)+O(1), t\right)\right| \mathrm{d} t \leq 3 M_{0} I_{0}^{\frac{\nu}{2}} \cdot \operatorname{Meas}\left[\Sigma_{1}\left(\theta_{0}\right)\right] \\
& \quad \leq 6 M_{0} I_{0}^{\frac{\nu}{2}} \arccos \left(\frac{2 I_{0}-\sqrt{a} \delta_{0}^{2}}{\sqrt{4 I_{0}^{2}-1}}\right) \\
& \quad \leq 6 M_{0} I_{0}^{\frac{\nu}{2}} \arccos \left(1-\frac{\sqrt{a} \delta_{0}^{2}}{2 I_{0}}\right) \rightarrow 0, \quad \text { as } I_{0} \rightarrow \infty .
\end{aligned}
$$

Therefore, we obtain that

$$
\lim _{I_{0} \rightarrow+\infty} \int_{\Sigma_{1}\left(\theta_{0}\right)} \varphi_{1}\left(\theta_{0}, I_{0}, t\right) p(t) \mathrm{d} t=0 .
$$

When $t \in \Sigma_{2}\left(\theta_{0}\right)$ and $I_{0}$ is large enough, we have $x\left(t ; \theta_{0}, I_{0}\right)+c_{0} \geq \delta_{0}$. Then

$$
\begin{aligned}
& \left|\int_{\Sigma_{1}\left(\theta_{0}\right)} \varphi_{1}\left(\theta_{0}, I_{0}, t\right) f\left(x\left(\theta_{0}, I_{0}\right)+O(1), t\right) \mathrm{d} t\right| \\
& \quad \leq \int_{\Sigma_{1}\left(\theta_{0}\right)}\left|\varphi_{1}\left(\theta_{0}, I_{0}, t\right) f\left(x\left(\theta_{0}, I_{0}\right)+O(1), t\right)\right| \mathrm{d} t \leq \frac{2 I_{0} M_{1}}{\sqrt{4 I_{0}^{2}-1}} \cdot \operatorname{Meas}\left[\Sigma_{2}\left(\theta_{0}\right)\right] \\
& \quad \leq 4 M_{1} \cdot \operatorname{Meas}\left[\Sigma_{2}\left(\theta_{0}\right)\right] \rightarrow 0, \text { as } I_{0} \rightarrow+\infty
\end{aligned}
$$

where $M_{1}$ is a constant defined in Lemma 4.2. Consequently, we obtain that

$$
\lim _{I_{0} \rightarrow+\infty} \int_{\Sigma_{2}\left(\theta_{0}\right)} \varphi_{1}\left(\theta_{0}, I_{0}, t\right) p(t) \mathrm{d} t=0 .
$$


Note that

$$
x\left(\theta_{0}, I_{0}\right)=a^{-1 / 4} \sqrt{2 I_{0}} \sqrt{1-\mathcal{K}\left(I_{0}\right) \cos \left(\theta_{0}+2 \sqrt{a} t\right)}+O(1), \quad I_{0} \rightarrow+\infty .
$$

For any $\theta_{0} \in[0,2 \pi]$, if $t \in \Sigma_{3}\left(\theta_{0}\right)$, then $\cos \left(\theta_{0}+2 \sqrt{a} t\right)<1-I_{0}^{-1 / 2} / 4$. Moreover, we have

$$
\begin{aligned}
x\left(\theta_{0}, I_{0}\right) & =a^{-1 / 4} \sqrt{2 I_{0}-\sqrt{4 I_{0}^{2}-1} \cos \left(\theta_{0}+2 \sqrt{a} t\right)}+O(1) \\
& \geq a^{-1 / 4} \sqrt{2 I_{0}-\sqrt{4 I_{0}^{2}-1}\left(1-I_{0}^{-1 / 2} / 4\right)}+O(1) \\
& \geq a^{-1 / 4} \sqrt{2 I_{0}-2 I_{0}\left(1-I_{0}^{-1 / 2} / 4\right)}+O(1) \\
& =\frac{\sqrt{2} a^{-1 / 4}}{2} I_{0}^{1 / 4}+O(1) \rightarrow+\infty, \quad \text { as } I_{0} \rightarrow+\infty .
\end{aligned}
$$

On the other hand, for any fixed $\theta_{0} \in \mathbb{R}, t \in \Sigma_{3}\left(\theta_{0}\right)$ and $I_{0}$ large enough, $\mathcal{K}\left(I_{0}\right)-\cos \left(\theta_{0}+2 \sqrt{a} t\right) \geq \mathcal{K}\left(I_{0}\right)-\left(1-I_{0}^{-1 / 2} / 4\right) \geq \mathcal{K}^{2}\left(I_{0}\right)-\left(1-I_{0}^{-1 / 2} / 4\right) \geq 0$, which implies that $\varphi_{1}\left(\theta_{0}, I_{0}, t\right) \geq 0$. Note that the limit

$$
\begin{aligned}
\lim _{I_{0}} \rightarrow+\infty & \varphi_{1}\left(\theta_{0}, I_{0}, t\right) \cdot f\left(x(\theta, I)+C_{0}, t\right) \\
& =\lim _{I_{0} \rightarrow+\infty} \frac{\mathcal{K}\left(I_{0}\right)-\cos \left(\theta_{0}+2 \sqrt{a} t\right)}{\sqrt{2} \mathcal{K}\left(I_{0}\right) \sqrt{1-\mathcal{K}\left(I_{0}\right) \cos \left(\theta_{0}+2 \sqrt{a} t\right)}} \cdot f\left(x\left(\theta_{0}, I_{0}\right)+O(1), t\right) \\
& =\lim _{\zeta \rightarrow 1-0} \frac{\zeta-\cos \left(\theta_{0}+2 \sqrt{a} t\right)}{\sqrt{2} \zeta \sqrt{1-\zeta \cos \left(\theta_{0}+2 \sqrt{a} t\right)}} \cdot f(+\infty, t)\left(\operatorname{let} \zeta=\mathcal{K}\left(I_{0}\right) .\right) \\
& =\sqrt{1-\cos \left(\theta_{0}+2 \sqrt{a} t\right)} \cdot f(+\infty, t)=\left|\sin \left(\theta_{0} / 2+\sqrt{a} t\right)\right| f(+\infty, t)
\end{aligned}
$$

holds uniformly for $t \in \Sigma_{3}\left(\theta_{0}\right)$, for any fixed $\theta_{0} \in \mathbb{R}$. In view of the boundedness of $\varphi_{1}\left(\theta_{0}, I_{0}, t\right) \cdot f\left(x(\theta, I)+c_{0}, t\right)$ on $t \in \Sigma_{3}\left(\theta_{0}\right)$, by Lebesgue's dominated convergence theorem, we have

$$
\begin{aligned}
\lim _{I_{0} \rightarrow+\infty} & \int_{\Sigma_{3}\left(\theta_{0}\right)} \varphi_{1}\left(\theta_{0}, I_{0}, t\right) \cdot f\left(x\left(\theta_{0}, I_{0}\right)+O(1), t\right) \mathrm{d} t \\
= & \lim _{I_{0} \rightarrow+\infty}\left(\int_{\Sigma_{3}\left(\theta_{0}\right)}-\int_{0}^{2 \pi}\right) \varphi_{1}\left(\theta_{0}, I_{0}, t\right) \cdot f\left(x\left(\theta_{0}, I_{0}\right)+O(1), t\right) \mathrm{d} t \\
& \quad+\lim _{I_{0} \rightarrow+\infty} \int_{0}^{2 \pi} \varphi_{1}\left(\theta_{0}, I_{0}, t\right) \cdot f\left(x\left(\theta_{0}, I_{0}\right)+O(1), t\right) \mathrm{d} t \\
= & \int_{0}^{2 \pi}\left|\sin \left(\theta_{0} / 2+\sqrt{a} t\right)\right| f(+\infty, t) \mathrm{d} t .
\end{aligned}
$$

Together with (4.17), (4.18) and (4.19), we have

$$
\begin{aligned}
\lim _{I_{0} \rightarrow+\infty} \psi_{1}\left(\theta_{0}, I_{0}\right) & =\lim _{I_{0} \rightarrow+\infty} \int_{0}^{2 \pi} \varphi_{1}\left(\theta_{0}, I_{0}, t\right) \cdot f\left(x\left(\theta_{0}, I_{0}\right)+O(1), t\right) \mathrm{d} t \\
& =\int_{0}^{2 \pi}\left|\sin \left(\theta_{0} / 2+\sqrt{a} t\right)\right| f(+\infty, t) \mathrm{d} t
\end{aligned}
$$


Thus, we have proved the first equality in Lemma 4.3. In the following, we will prove the second equality.

For any $\theta_{0} \in \mathbb{R}, t \in \Sigma_{1}\left(\theta_{0}\right)$ and $I_{0}$ is large enough, from the proof of Lemma 4.2, we know that

$$
\begin{aligned}
& \left|\varphi_{2}\left(\theta_{0}, I_{0}, t\right) \cdot f\left(x\left(\theta_{0}, I_{0}\right)+O(1), t\right)\right| \\
& \quad \leq\left|\frac{\mathcal{K}\left(I_{0}\right) \sin \left(\theta_{0}+2 \sqrt{a} t\right)}{\sqrt{2} \sqrt{1-\mathcal{K}\left(I_{0}\right) \cos \left(\theta_{0}+2 \sqrt{a} t\right)}}\right| \cdot \frac{M_{0}}{\left(\sqrt{2 I_{0}-\sqrt{4 I_{0}^{2}-1} \cos \theta_{0}}+O(1)\right)^{\nu}} \\
& \quad \leq\left|\frac{\sqrt{4 I_{0}^{2}-1} \sin \left(\theta_{0}+2 \sqrt{a} t\right)}{2 \sqrt{I_{0}} \cdot \sqrt{2 I_{0}-\sqrt{4 I_{0}^{2}-1} \cos \left(\theta_{0}+2 \sqrt{a} t\right)}}\right| \cdot M_{0} I_{0}^{\frac{\nu}{2}} \leq M_{0} I_{0}^{\frac{\nu}{2}} .
\end{aligned}
$$

Similarly,

$$
\begin{aligned}
& \left|\int_{\Sigma_{1}\left(\theta_{0}\right)} \varphi_{2}\left(\theta_{0}, I_{0}, t\right) f\left(x\left(\theta_{0}, I_{0}\right)+O(1), t\right) \mathrm{d} t\right| \leq \int_{\Sigma_{1}\left(\theta_{0}\right)}\left|\varphi_{2}\left(\theta_{0}, I_{0}, t\right) f(\cdot, t)\right| \mathrm{d} t \\
& \quad \leq M_{0} I_{0}^{\frac{\nu}{2}} \cdot \operatorname{Meas}\left[\Sigma_{1}\left(\theta_{0}\right)\right] \rightarrow 0, \quad \text { as } I_{0} \rightarrow+\infty,
\end{aligned}
$$

which leads to

$$
\lim _{I_{0} \rightarrow+\infty} \int_{\Sigma_{1}\left(\theta_{0}\right)} \varphi_{2}\left(\theta_{0}, I_{0}, t\right) f\left(x\left(\theta_{0}, I_{0}\right)+O(1), t\right) \mathrm{d} t=0 .
$$

Recall $x(\theta, I)+c_{0}>\delta_{0}$ for all $t \in \Sigma_{2}\left(\theta_{0}\right)$. With the same argument, for $I_{0} \rightarrow+\infty$,

$$
\left|\int_{\Sigma_{2}\left(\theta_{0}\right)} \varphi_{2}\left(\theta_{0}, I_{0}, t\right) f\left(x\left(\theta_{0}, I_{0}\right)+O(1), t\right) \mathrm{d} t\right| \leq M_{1} \cdot \operatorname{Meas}\left[\Sigma_{2}\left(\theta_{0}\right)\right] \rightarrow 0 .
$$

Then we have

$$
\lim _{I_{0} \rightarrow+\infty} \int_{\Sigma_{2}\left(\theta_{0}\right)} \varphi_{2}\left(\theta_{0}, I_{0}, t\right) f\left(x\left(\theta_{0}, I_{0}\right)+O(1), t\right) \mathrm{d} t=0 .
$$

For any fixed $\theta_{0} \in \mathbb{R}$, the limit

$$
\begin{aligned}
& \lim _{I_{0} \rightarrow+\infty} \varphi_{2}\left(\theta_{0}, I_{0}, t\right) \cdot f\left(x\left(\theta_{0}, I_{0}\right)+O(1), t\right) \\
& =\lim _{I_{0} \rightarrow+\infty} \frac{\mathcal{K}\left(I_{0}\right) \sin \left(\theta_{0}+2 \sqrt{a} t\right)}{\sqrt{2} \sqrt{1-\mathcal{K}\left(I_{0}\right) \cos \left(\theta_{0}+2 \sqrt{a} t\right)}} \cdot f\left(x\left(\theta_{0}, I_{0}\right)+O(1), t\right) \\
& =\lim _{\zeta \rightarrow 1-0} \frac{\zeta \sin \left(\theta_{0}+2 \sqrt{a} t\right)}{\sqrt{2} \sqrt{1-\zeta \cos \left(\theta_{0}+2 \sqrt{a} t\right)}} \cdot f(+\infty, t)\left(\operatorname{let} \zeta=\mathcal{K}\left(I_{0}\right) .\right) \\
& =\frac{\sin \left(\theta_{0}+2 \sqrt{a} t\right)}{\sqrt{1-\cos \left(\theta_{0}+2 \sqrt{a} t\right)}} \cdot f(+\infty, t) \\
& =\operatorname{sign}\left(\sin \left(\theta_{0} / 2+\sqrt{a} t\right)\right) \cdot \cos \left(\theta_{0} / 2+\sqrt{a} t\right) f(+\infty, t)
\end{aligned}
$$


holds uniformly for $t \in \Sigma_{3}\left(\theta_{0}\right)$. Therefore, by Lebesgue's dominated convergence theorem, we have

$$
\begin{aligned}
\lim _{I_{0} \rightarrow+\infty} \psi_{2}\left(\theta_{0}, I_{0}\right) & =\lim _{I_{0} \rightarrow+\infty} \int_{\Sigma_{3}\left(\theta_{0}\right)} \varphi_{2}\left(\theta_{0}, I_{0}, t\right) \cdot f\left(x\left(\theta_{0}, I_{0}\right)+O(1), t\right) \mathrm{d} t \\
& =\int_{0}^{2 \pi} \operatorname{sign}\left(\sin \left(\theta_{0} / 2+\sqrt{a} t\right)\right) \cdot \cos \left(\theta_{0} / 2+\sqrt{a} t\right) f(+\infty, t) \mathrm{d} t .
\end{aligned}
$$

Combined with (4.20) and (4.21), we have proved (4.8).

Lemma 4.5. Assume that the continuous function $f:\left(-c_{0},+\infty\right) \times[0,2 \pi] \rightarrow \mathbb{R}$ satisfies $\left(f_{1}\right)$ and $\left(f_{3}\right)$. Then for $I_{0} \rightarrow+\infty$, we have that

$$
\int_{0}^{2 \pi} O\left(I_{0}^{-1}\right) f\left(x\left(\theta_{0}, I_{0}\right)+O(1), t\right) \mathrm{d} t=O\left(I_{0}^{-1}\right)
$$

and

$$
\int_{0}^{2 \pi} O\left(I_{0}^{-\frac{1}{2}}\right) f\left(x\left(\theta_{0}, I_{0}\right)+O(1), t\right) \mathrm{d} t=O\left(I_{0}^{-\frac{1}{2}}\right),
$$

uniformly with respect to $\theta_{0} \in[0,2 \pi]$.

Proof. The proof of Lemma 4.5 is similar to the proof of Lemma 4.4. Notice that

$$
\begin{aligned}
& \left|\int_{0}^{2 \pi} O\left(I_{0}^{-1}\right) f\left(x\left(\theta_{0}, I_{0}\right)+O(1), t\right) \mathrm{d} t\right| \\
& \quad=\left|\left(\int_{\Sigma_{1}\left(\theta_{0}\right)}+\int_{[0,2 \pi] \backslash \Sigma_{1}\left(\theta_{0}\right)}\right) O\left(I_{0}^{-1}\right) f\left(x\left(\theta_{0}, I_{0}\right)+O(1), t\right) \mathrm{d} t\right| \\
& \quad \leq I_{0}^{\frac{\nu}{2}} \cdot \operatorname{Meas}\left[\Sigma_{1}\left(\theta_{0}\right)\right] \cdot O\left(I_{0}^{-1}\right)+M_{1} \cdot O\left(I_{0}^{-1}\right)=O\left(I_{0}^{-1}\right), \quad \text { as } I_{0} \rightarrow+\infty .
\end{aligned}
$$

Therefor, we obtain the desired (4.22), and (4.23) can be proved similarly.

Set $r_{0}=I_{0}^{\frac{1}{2}}$ and

$$
\sigma_{f}(\theta)=\int_{0}^{2 \pi}|\sin (\theta / 2+\sqrt{a} t)| f(+\infty, t) \mathrm{d} t,
$$

then we have

$$
\sigma_{f}^{\prime}(\theta)=\frac{1}{2} \int_{0}^{2 \pi} \operatorname{sign}(\sin (\theta / 2+\sqrt{a} t)) \cdot \cos (\theta / 2+\sqrt{a} t) f(+\infty, t) \mathrm{d} t .
$$

By (4.16) and Lemma 4.4 and Lemma 4.5 we get that

Lemma 4.6. Assume that the continuous function $f:\left(-c_{0},+\infty\right) \times[0,2 \pi] \rightarrow \mathbb{R}$ satisfies $\left(f_{1}\right)$ and $\left(f_{2}\right)$. Then for $r_{0} \rightarrow+\infty$, we have the mapping $\mathcal{P}_{1}$ as

$$
\left\{\begin{array}{l}
\theta_{1}=\theta_{0}+4 \sqrt{a} \pi+a^{-1 / 4} r_{0}^{-1} \sigma_{f}\left(\theta_{0}\right)+H\left(\theta_{0}, r_{0}\right) \\
r_{1}=r_{0}-a^{-1 / 4} \sigma_{f}^{\prime}\left(\theta_{0}\right)+G\left(\theta_{0}, r_{0}\right)
\end{array}\right.
$$

where $H\left(\theta_{0}, r_{0}\right), G\left(\theta_{0}, r_{0}\right) \in C[\mathbb{R} / 2 \pi \mathbb{Z} \times(1,+\infty), \mathbb{R}]$ are $2 \pi$-periodic with respect to $\theta_{0}$ such that $H\left(\theta_{0}, r_{0}\right)=o\left(r_{0}^{-1}\right)$ and $G\left(\theta_{0}, r_{0}\right)=o(1)$. 


\section{The proofs of Theorem 1.1 and Theorem 2.1}

\subsection{Proof of Theorem 2.1}

First, we proof the existence of $2 \pi$-periodic solutions. If $\sigma_{f}(\theta) \neq 0, \theta \in[0,2 \pi]$, then $\sigma_{f}(\theta)$ has a constant sign, which implies that there exists a positive constant $d$ such that $\sigma_{f}(\theta)>d$ or $\sigma_{f}(\theta)<-d$ for any $\theta \in[0,2 \pi]$. By Lemma 4.5, for $r_{0}$ large enough, we have $\left|\theta_{1}-\theta_{0}-2 \sqrt{a} \pi\right|<1$. So it follows that the image $\left(\theta_{1}, r_{1}\right)$ can never lie on the ray $\theta_{1}=\theta_{0}$. Hence, by Poincaré-Bohl theorem [29], the mapping $\mathcal{P}_{1}$ has at least one fixed point and therefore the Poincaré mapping $P$ has at least one fixed point, which implies the existence of $2 \pi$-periodic solutions.

In the following, we will prove the first part for existence of unbounded solutions. By Proposition 2.1 of [23], if $\sigma_{f}^{\prime}(\theta)>0$ and for all $\theta \in[0,2 \pi]$, $\left|\sigma_{f}(\theta)\right|+\left|\sigma_{f}^{\prime}(\theta)\right|>0$, we know that there exists $R_{0}>0$ such that, if $r_{0} \geq R_{0}$, then the orbit $\left\{\left(\theta_{j}, r_{j}\right)\right\}$ exists in the future and satisfies $\lim _{j \rightarrow+\infty} r_{j}=+\infty$.

Then for $I_{0}$ large enough, the orbit $\left\{\left(\theta_{j}, I_{j}\right)\right\}$ exists in the future and satisfies $\lim _{j \rightarrow+\infty} I_{j}=+\infty$. So it follows that $\lim _{t \rightarrow+\infty} I(t)=+\infty$ by the conservation of energy. In view of (3.3) and (4.16), we get

$$
\lim _{t \rightarrow+\infty}\left(y(t)^{2}+x(t)^{2}+x(t)^{-2}\right)=+\infty .
$$

If for all $t \in\left[t_{0},+\infty\right)$,

$$
\limsup _{t \rightarrow+\infty}|x(t)| \leq d<+\infty
$$

then we get

$$
\lim _{t \rightarrow+\infty}\left(y(t)^{2}+x(t)^{-2}\right)=+\infty .
$$

Let us define the positive function

$$
J(t)=y(t)^{2}+x(t)^{-2}
$$

and we have

$$
\begin{aligned}
\left|\frac{\mathrm{d} J}{\mathrm{~d} t}\right| & =\left|2 y(t) y^{\prime}(t)-2 x(t)^{-3} x^{\prime}(t)\right| \\
& =|-2 a x(t) y(t)+2 y(t)(p(t)-g(x))| \\
& \leq C_{3} J(t) .
\end{aligned}
$$

Using the Gronwall's inequality, we have $J(t) \leq J(0)$, which contradicts (5.1). The case $\sigma_{f}^{\prime}(\theta)<0$ can be handled in a similar way.

\subsection{Proof of Theorem 1.1}

Now, we consider the existence of periodic solutions of (2.9) under the assumption $\left(f_{2}\right)$. Rewrite Eq. (2.9) in the following equivalent form

$$
x^{\prime \prime}(t)-\frac{1+\mu^{2}}{x^{3}(t)}+a x(t)+\bar{f}(x(t), t)=0,
$$

where $\bar{f}(x(t), t)=f(x(t), t)+\frac{1}{x^{3}}$. 
We can verify that $\bar{f}$ satisfies $\left(f_{1}\right)$ and $\left(f_{3}\right)$. For $\mu \in[0,1]$, we can rescale Eq. (5.2) as

$$
x^{\prime \prime}(t)-\frac{1}{x^{3}(t)}+a x(t)+\frac{\bar{f}(x(t), t)}{\left(1+\mu^{2}\right)^{1 / 4}}=0 .
$$

With similar arguments for Eq. (2.9), we obtain a family of Poincaré mappings $\mathcal{P}(z, \mu)$ with the parameter $\mu \in[0,1]$, whose generalized polar expression is written in the following form

$$
\left\{\begin{array}{l}
\theta_{1}=\theta_{0}+4 \sqrt{a} \pi+a^{-1 / 4} r_{0}^{-1} \sigma_{\bar{f}}\left(\theta_{0}\right)+H\left(\theta_{0}, r_{0} ; \mu\right), \\
r_{1}=r_{0}-a^{-1 / 4} \sigma_{\bar{f}}^{\prime}\left(\theta_{0}\right)+G\left(\theta_{0}, r_{0} ; \mu\right),
\end{array}\right.
$$

where $H\left(\theta_{0}, r_{0} ; \mu\right), G\left(\theta_{0}, r_{0} ; \mu\right) \in C[\mathbb{R} / 2 \pi \mathbb{Z} \times(1,+\infty) \times[0,1], \mathbb{R}]$ are $2 \pi$-periodic with respect to $\theta_{0}$ such that $H\left(\theta_{0}, r_{0} ; \mu\right)=o\left(r_{0}^{-1}\right)$ and $G\left(\theta_{0}, r_{0} ; \mu\right)=o(1)$ as $r_{0} \rightarrow+\infty$ uniformly for $\mu \in[0,1]$.

Let $B_{r_{0}}$ be a open and bound ball with radius $r_{0}$ and center at the origin $O:$

$$
B_{r_{0}}=\left\{|z|<r_{0}\right\} \text {, }
$$

and let $\Omega=B_{r_{0}} \times[0,1], \Omega_{\mu}=\{z:(z, \mu) \in \Omega\}$. Let

$$
\Sigma=\{(z, \mu) \in \bar{\Omega}: z-\mathcal{P}(z, \mu)=0\},
$$

and for $\mu \in[0,1]$, let $\Sigma_{\mu}=\{z:(z, \mu) \in \Sigma\}$. Consider the operator equation

$$
z-\mathcal{P}(z, \mu)=0, \quad(z, \mu) \in \bar{\Omega} .
$$

Clearly, $\mathcal{P}$ is a continuous operator on $\bar{\Omega}$. If $\sigma_{\bar{f}}(\theta) \neq 0$, for any $\theta \in[0,2 \pi]$, we know that, for any $(z, \mu) \in \partial \Omega$, we have $z \neq \mathcal{P}(z, \mu)$ as $r_{0}$ large enough. Moreover, the polar image $\left(\theta_{1}, r_{1}\right)$ of $\mathcal{P}(z, \mu)$ can never lie on the ray $\theta=\theta_{0}$. Then Poincaré-Bohl theorem [29] allows one to conclude that

$$
\operatorname{deg}\left(\mathcal{P}, \Omega_{0}, O\right)=\operatorname{deg}\left(\mathcal{I}_{d}, \Omega_{0}, O\right)=1 .
$$

Using the continuation lemma $[30, \S 4.4]$, there exists a closed connected subset $\Sigma^{*}$ of $\Sigma$ joining $\Sigma_{0} \times\{0\}$ to $\Sigma_{1} \times\{1\}$. Note that every fixed point $z_{\mu}$ of the Poincaré map $\mathcal{P}$ corresponds to the periodic solution $x\left(t ; \theta_{0}, r_{0}, \mu\right)$ of $(5.3)$. Consequently, by the continuation of the solution with respect to the initial value, we have obtained a closed connected set of $2 \pi$-periodic solutions

$$
\mathcal{C}=\{(\mu, x): \mu \in[0,1], x(t ; \mu) \text { is } 2 \pi \text {-periodic and satisfies equation }(5.2)\}
$$

such that $\mathcal{C} \subset[0,1] \times C_{T}$.

Now defining

$$
\varphi(t)=\int_{0}^{t} \frac{\mu}{x^{2}(s ; \mu)} \mathrm{d} s,
$$

we know that Eq. (2.10) is satisfied and

$$
\begin{aligned}
\frac{\varphi(t+2 \pi)-\varphi(t)}{2 \pi} & =\frac{1}{2 \pi} \int_{t}^{t+2 \pi} \frac{\mu}{x^{2}(t ; \mu)} \mathrm{d} t \\
& =\frac{1}{2 \pi} \int_{0}^{2 \pi} \frac{\mu}{x^{2}(t ; \mu)} \mathrm{d} t=\vartheta(\mu) .
\end{aligned}
$$


Note that

$$
\tilde{\varphi}(t)=\int_{0}^{t}\left[\frac{\mu}{x^{2}(t ; \mu)}-\vartheta(\mu)\right] \mathrm{d} t=\varphi(t)-\vartheta t
$$

is $2 \pi$-periodic, and

$$
\mathbf{u}(t)=x(t)(\cos (\tilde{\varphi}(t)+\vartheta t), \sin (\tilde{\varphi}(t)+\vartheta t)) .
$$

Consider $\mu \in[0,1]$, and we know that $\vartheta(0)=0$ and $\vartheta(1)>0$. Therefore, there exists constants $\mu_{1}, \mu_{2}$ being close to zero such that, $0<\mu_{1}<\mu_{2}$ and

$$
\min _{\mu \in\left[\mu_{1}, \mu_{2}\right]} \vartheta(\mu)<\max _{\mu \in\left[\mu_{1}, \mu_{2}\right]} \vartheta(\mu)
$$

Now for any $\vartheta\left(\mu_{q p}\right)=\frac{q}{p} \in\left[\min _{\mu \in\left[\mu_{1}, \mu_{2}\right]} \vartheta(\mu), \max _{\mu \in\left[\mu_{1}, \mu_{2}\right]} \vartheta(\mu)\right]$ with $(p, q)=1$ and $\mu_{q p} \in\left[\mu_{1}, \mu_{2}\right]$, then $\mathbf{u}_{\mu_{q p}}(t)$ is periodic with minimal period $2 p \pi$, and rotates exactly $p$ times around the origin in the period time $2 p \pi$. For any $\omega \in\left[\min _{\mu \in\left[\mu_{1}, \mu_{2}\right]} \vartheta(\mu), \max _{\mu \in\left[\mu_{1}, \mu_{2}\right]} \vartheta(\mu)\right] \backslash \mathbb{Q}, \mathbf{u}_{\omega}(t)$ is a quasi-periodic with the frequencies $\langle 1, \omega\rangle$. We also recall

$$
\mathbf{u}(t)=x_{\mu}(t) e^{i \varphi(t)} .
$$

is a radially $2 \pi$-periodic solution of the original system (1.1). Since $x(t ; \mu)$ is continuous on $[0,2 \pi] \times\left[\mu_{1}, \mu_{2}\right]$, we obtain (1.8) while taking

$$
B\left(\mu_{1}, \mu_{2}\right)=\max _{(t, \mu) \in[0,2 \pi] \times\left[\mu_{1}, \mu_{2}\right]}|x(t ; \mu)| .
$$

The second part of the proof follows from Theorem 2.1 directly. Thus we end the proof.

\section{Acknowledgments}

This work is partially supported by the National Natural Science Foundation of China (Grant Nos. 11226130, 11301106, 11271277) and by Spanish MICINN Grant with FEDER funds MTM2011-23652.

\section{Appendix A}

Lemma 5.1. Assume that $\Gamma$ is a simple closed curve on the plane defined by

$$
\Gamma: \frac{1}{2} y^{2}+\frac{1}{2} a\left(x+c_{0}\right)^{2}+\frac{1}{2\left(x+c_{0}\right)^{2}}=\hat{h}+\sqrt{a}(\hat{h} \geq 0) .
$$

Then the area enclosed by $\Gamma$ in phase space swept out in one period/ $2 \pi$ is given by

$$
I(\hat{h})=\frac{1}{2 \pi} \oint_{\Gamma} y \mathrm{~d} x=\frac{\hat{h}}{2 \sqrt{a}} .
$$


Proof. Notice that $x+c_{0}>0$. By the definition of the curve $\Gamma$, we have

$$
\sqrt{a}\left(x+c_{0}\right)+\frac{1}{x+c_{0}}=\sqrt{2(\hat{h}+2 \sqrt{a})-y^{2}} .
$$

It follows that

$$
x(y)=\frac{1}{2 \sqrt{a}}\left(\sqrt{2(\hat{h}+2 \sqrt{a})-y^{2}} \pm \sqrt{2 \hat{h}-y^{2}}\right)-c_{0} .
$$

So we divide the curve $\Gamma$ into two parts

$$
\begin{aligned}
& x_{1}(y)=\frac{1}{2 \sqrt{a}}\left(\sqrt{2(\hat{h}+2 \sqrt{a})-y^{2}}-\sqrt{2 \hat{h}-y^{2}}\right)-c_{0},-\sqrt{2 \hat{h}} \leq y \leq \sqrt{2 \hat{h}}, \\
& x_{2}(y)=\frac{1}{2 \sqrt{a}}\left(\sqrt{2(\hat{h}+2 \sqrt{a})-y^{2}}+\sqrt{2 \hat{h}-y^{2}}\right)-c_{0},-\sqrt{2 \hat{h}} \leq y \leq \sqrt{2 \hat{h}},
\end{aligned}
$$

as shown is Fig. 1. Using the Green formula, We have

$$
\begin{aligned}
I(\hat{h}) & =\frac{1}{2 \pi} \oint_{\Gamma} y \mathrm{~d} x=\frac{1}{2 \pi} \iint_{D} \mathrm{~d} x \mathrm{~d} y=\frac{1}{2 \pi} \int_{-\sqrt{2 \hat{h}}}^{\sqrt{2 \hat{h}}}\left(x_{2}(y)-x_{1}(y)\right) \mathrm{d} y \\
& =\frac{1}{2 \sqrt{a} \pi} \int_{-\sqrt{2 h}}^{\sqrt{2 h}} \sqrt{2 \hat{h}-y^{2}} \mathrm{~d} y=\frac{1}{2 \sqrt{a} \pi} \cdot \frac{1}{2} \pi(\sqrt{2 \hat{h}})^{2}=\frac{\hat{h}}{2 \sqrt{a}},
\end{aligned}
$$

where $D$ is the domain enclosed by $\Gamma$ and we take $\Gamma$ the negative direction along the boundary of $D$.

Lemma 5.2. Assume that

$$
f(x)=\frac{(b-1)(1-x)}{[b-(b-1) x]^{n}}, \quad n \geq 1,
$$

where $b>1$ is a positive constant, then

$$
|f(x)| \leqslant \max \left\{\frac{(n-1)^{n+1}}{n^{n}}, \frac{(b-1)(1-\lambda)}{[b-(b-1) \lambda]^{n}}\right\},
$$

for $x \in[\lambda, 1]$ and $0<\lambda<1$.

Proof. The function has the minimum and maximum values for $x \in[\lambda, 1]$, since it is continuous on the closed interval. Note that, if $n>1$, then

$$
f^{\prime}(x)=\frac{b-1}{[b-(b-1) x]^{n+1}}[(n-1) b-n-(n-1)(b-1) x] .
$$

It follows that the function $f$ has a stationary point

$$
x_{0}=\frac{(n-1) b-n}{(n-1) b-(n-1)} \text {. }
$$

Moreover, we have

$$
f\left(x_{0}\right)=\frac{(n-1)^{n+1}}{n^{n}}, \quad f(1)=0
$$

and 


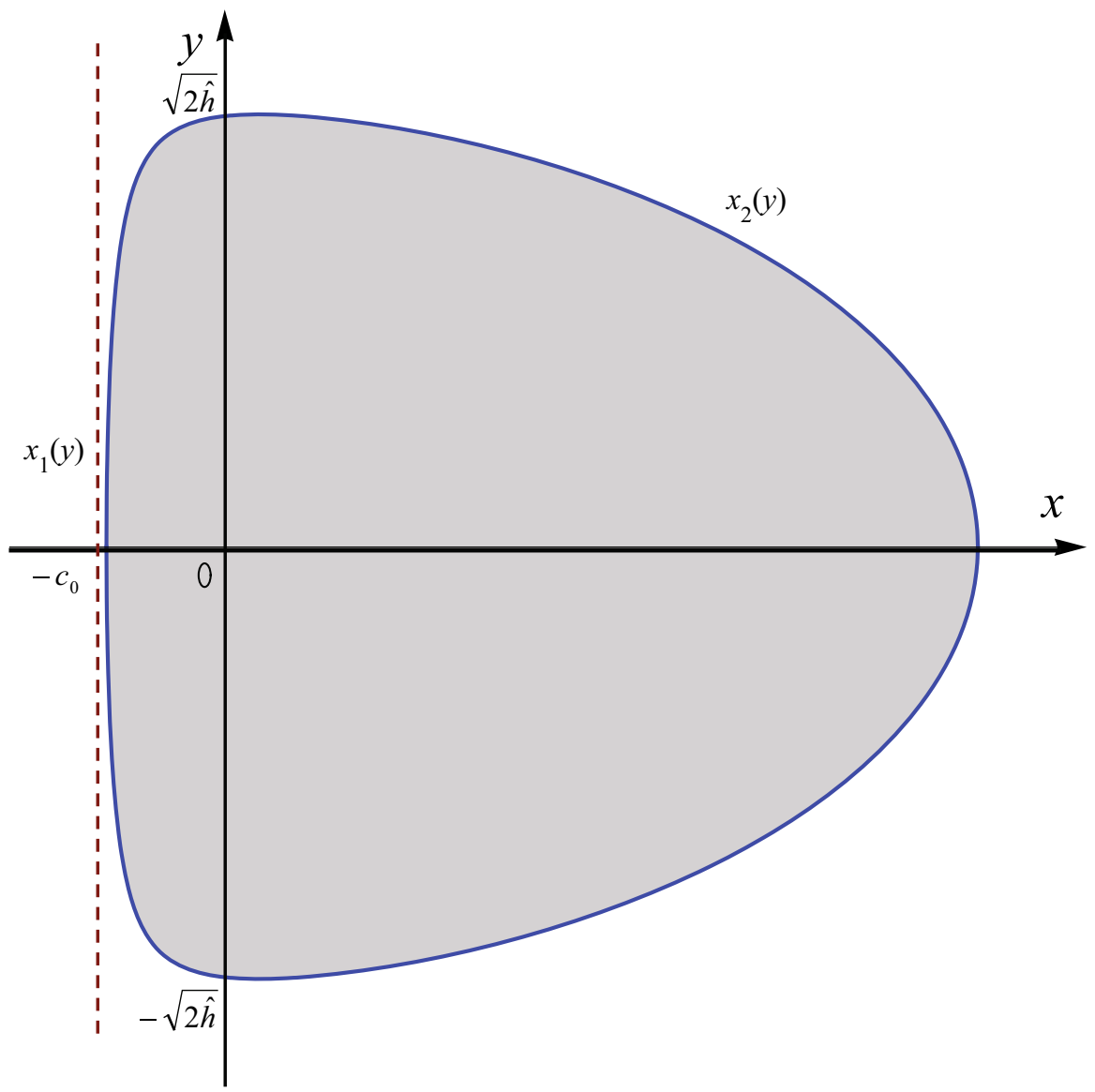

Figure 1. The area enclosed by $\Gamma$ in phase space swept out in one period $/ 2 \pi$

$$
f(\lambda)=\frac{(b-1)(1-\lambda)}{[b-(b-1) \lambda]^{n}} .
$$

Therefore, for $x \in[\lambda, 1]$, we get that

$$
0 \leq f(x) \leq \max \left\{f\left(x_{0}\right), f(1), f(\lambda)\right\}
$$

In case of $n=1$, we have $f^{\prime}(x)<0$. Also for $x \in[\lambda, 1]$, we get that

$$
0 \leq f(x) \leq \max \{f(1), f(\lambda)\}
$$

Thus the proof is finished. 


\section{Appendix B: Calculations of (3.4), (3.8) and (3.9)}

\section{B.1. Calculation of (3.4)}

Note that $\xi=\left(\tau+c_{0}\right)^{2}$ is a monotone increasing function on the interval $t \in\left(x_{-}, x\right)$. Then we have

$$
\begin{aligned}
\int_{x_{-}}^{x} & \frac{2 \sqrt{a}}{\sqrt{2(h+\sqrt{a})-a\left(\tau+c_{0}\right)^{2}-\left(\tau+c_{0}\right)^{-2}}} \mathrm{~d} \tau \\
& =\int_{\left(x_{-}+c_{0}\right)^{2}}^{\left(x+c_{0}\right)^{2}} \frac{2 \sqrt{a}}{\sqrt{2(h+\sqrt{a}) \xi-a \xi^{2}-1}} \mathrm{~d} \xi \quad\left(\text { let } \xi=\left(\tau+c_{0}\right)^{2}\right) \\
& =\left[\arcsin \frac{\sqrt{a} \xi-\frac{h+\sqrt{a}}{\sqrt{a}}}{\sqrt{\frac{h^{2}}{a}+\frac{2 h}{\sqrt{a}}}}\right]_{\left(x_{-}+c_{0}\right)^{2}}^{\left(x+c_{0}\right)^{2}} \\
& =\frac{\pi}{2}-\arcsin \frac{h+\sqrt{a}-a\left(x+c_{0}\right)^{2}}{\sqrt{h^{2}+2 \sqrt{a} h}} .
\end{aligned}
$$

\section{B.2. Calculations of (3.8) and (3.9)}

By (3.4), we know that

$$
\cos \theta=\sin (\pi / 2-\theta)=\frac{h+\sqrt{a}-a\left(x+c_{0}\right)^{2}}{\sqrt{h^{2}+2 \sqrt{a} h}} .
$$

From (3.4), we have

$$
\cos \theta=\frac{2 \sqrt{a} \tilde{I}+\sqrt{a}-a\left(x+c_{0}\right)^{2}}{\sqrt{4 a \tilde{I}^{2}+4 a \tilde{I}}} .
$$

Note that $x+c_{0}>0$. Then we have

$$
x=\frac{1}{a^{1 / 4}} \sqrt{2 \tilde{I}+1+\sqrt{4 \tilde{I}^{2}+4 \tilde{I}} \cos \theta}-c_{0} .
$$

Substituting $I=\tilde{I}-\frac{1}{2}$ into the equality above, we obtain the desired equality (3.8). On the other hand, in view of (3.8), we get

$$
\left(x+c_{0}\right)^{2}=\frac{1}{a^{1 / 2}}\left(2 I-\sqrt{4 I^{2}-1} \cos \theta\right) .
$$

Substituting it into (3.6), together with (3.5), we obtain the desired equality $(3.9)$.

\section{References}

[1] Fonda, A., Manásevich, R., Zanolin, F.: Subharmonic solutions for some secondorder differential equations with singularities. SIAM J. Math. Anal. 24(5), 1294-1311 (1993)

[2] Chu, J., Torres, P.J., Zhang, M.: Periodic solutions of second order nonautonomous singular dynamical systems. J. Differ. Equ. 239(1), 196-212 (2007) 
[3] Franco, D., Torres, P.: Periodic solutions of singular systems without the strong force condition. Proc. Am. Math. Soc. 136(4), 1229-1236 (2008)

[4] Fonda, A., Toader, R.: Periodic orbits of radially symmetric Keplerian-like systems: a topological degree approach. J. Differ. Equ. 244(12), 3235-3264 (2008)

[5] Qian, D., Torres, P.J.: Bouncing solutions of an equation with attractive singularity. Proc. R. Soc. Edinb. A Math. 134(1), 201-214 (2004)

[6] Capietto, A., Dambrosio, W., Liu, B.: On the boundedness of solutions to a nonlinear singular oscillator. Zeitschrift für Angewandte Mathematik Und Physik 60(6), 1007-1034 (2009)

[7] Liu, B.: Quasi-periodic solutions of forced isochronous oscillators at resonance. J. Differ. Equ. 246(9), 3471-3495 (2009)

[8] Torres, P.J.: Mathematical Models with Singularities. Atlantis Briefs in Differential Equations, vol. 1. Atlantis Press (2015)

[9] Fonda, A., Toader, R.: Radially symmetric systems with a singularity and asymptotically linear growth. Nonlinear Anal. Theory Methods Appl. 74(7), 2485-2496 (2011)

[10] Fonda, A., Toader, R.: Periodic orbits of radially symmetric systems with a singularity: the repulsive case. Adv. Nonlinear Stud. 11, 853-874 (2011)

[11] Fonda, A., Ureña, A.J.: Periodic, subharmonic, and quasi-periodic oscillations under the action of a central force. Discrete Contin. Dyn. Syst. 29, 169-192 (2011)

[12] Fonda, A., Toader, R.: Periodic solutions of radially symmetric perturbations of Newtonian systems. Proc. Am. Math. Soc. 140(4), 1331-1341 (2012)

[13] Fonda, A., Toader, R., Zanolin, F.: Periodic solutions of singular radially symmetric systems with superlinear growth. Annali di Matematica Pura ed Applicata 191(2), 181-204 (2012)

[14] Arnol'd, V.I.: Mathematical Methods of Classical Mechanics, vol. 60. Springer, Berlin (1989)

[15] Bolotin, S., MacKay, R.S.: Isochronous potentials. In: Localization and Energy Transfer in Nonlinear Systems. World Scientific Publishing Company, Singapore (2003)

[16] Fonda, A., Toader, R.: Periodic solutions of radially symmetric perturbations of Newtonian systems. Proc. Am. Math. Soc. 140(4), 1331-1341 (2012)

[17] Fonda, A., Ureña, A.J.: Periodic, subharmonic, and quasi-periodic oscillations under the action of a central force. Discrete Contin. Dyn. Syst. (DCDS-A) 29, 169-192 (2011)

[18] Liu, Q., Qian, D.: Modulated amplitude waves with nonzero phases in Bose -Einstein condensates. J. Math. Phys. 52, 082702 (2011) 
[19] Bonheure, D., Fabry, C., Smets, D.: Periodic solutions of forced isochronous oscillators at resonance. Discrete Contin. Dyn. Syst. 8(4), 907-930 (2002)

[20] Bonheure, D., Fabry, C.: Unbounded solutions of forced isochronous oscillators at resonance. Differ. Integral Equ. 15(9), 1139-1152 (2002)

[21] Bonheure, D., Fabry, C., Smets, D.: On a class of forced nonlinear oscillators at resonance. Equadiff 10, 37-43 (2002)

[22] Alonso, J.M., Ortega, R.: Unbounded solutions of semilinear equations at resonance. Nonlinearity 9(5), 1099-1112 (1996)

[23] Alonso, J.M., Ortega, R.: Roots of unity and unbounded motions of an asymmetric oscillator. J. Differ. Equ. 143(1), 201-220 (1998)

[24] Capietto, A., Dambrosio, W., Wang, Z.: Coexistence of unbounded and periodic solutions to perturbed damped isochronous oscillators at resonance. Proc. R. Soc. Edinb. Sect. A Math. 138(1), 15 (2008)

[25] Li, X., Zhang, Z.: Unbounded solutions and periodic solutions for second order differential equations with asymmetric nonlinearity. Proc. Am. Math. Soc., pp. 2769-2777 (2007)

[26] Ortega, R.: Boundedness in a piecewise linear oscillator and a variant of the small twist theorem. Proc. Lond. Math. Soc. 79(02), 381-413 (1999)

[27] Wang, Z.: Irrational rotation numbers and unboundedness of solutions of the second order differential equations with asymmetric nonlinearities. Proc. Am. Math. Soc., pp. 523-531 (2003)

[28] Wang, Z.: Coexistence of unbounded solutions and periodic solutions of Liénard equations with asymmetric nonlinearities at resonance. Sci. China Ser. A: Math. 50(8), 1205-1216 (2007)

[29] Lloyd, N.G.: Degree Theory, vol. 279. Cambridge University Press, Cambridge (1978)

[30] Mawhin, J.: Topological Fixed Point Theory and Nonlinear Differential Equations. Handbook of Topological Fixed Point Theory. Springer, Berlin (2005)

Qihuai Liu

School of Mathematical Sciences

Fudan University

Shanghai 200433

People's Republic of China

e-mail: qhuailiu@gmail.com

Qihuai Liu

School of Mathematics and Computing Science

Guilin University of Electronic Technology

Guilin 541003

People's Republic of China 
Pedro J. Torres

Departamento de Matemáptica Aplicada

Facultad de Ciencias

Universidad de Granada

18071 Granada

Spain

e-mail: ptorres@ugr.es

Dingbian Qian

School of Mathematical Sciences

Soochow University

Suzhou 215006

People's Republic of China

e-mail: dbqian@suda.edu.cn

Received: 17 July 2014.

Accepted: 16 February 2015. 